\title{
Osteopontin mediates glioblastoma-associated macrophage infiltration and is a potential therapeutic target
}

\author{
Jun Wei, ${ }^{1}$ Anantha Marisetty, ${ }^{1}$ Brett Schrand, ${ }^{2}$ Konrad Gabrusiewicz, ${ }^{1}$ Yuuri Hashimoto, ${ }^{1}$ Martina Ott, ${ }^{1}$ Zacharia Grami, ${ }^{1}$ \\ Ling-Yuan Kong, ${ }^{1}$ Xiaoyang Ling, ${ }^{1}$ Hillary Caruso, ${ }^{1}$ Shouhao Zhou, ${ }^{3}$ Y. Alan Wang, ${ }^{4}$ Gregory N. Fuller, ${ }^{5}$ Jason Huse, ${ }^{5}$ Eli Gilboa, ${ }^{2}$ \\ Nannan Kang, ${ }^{6}$ Xingxu Huang, ${ }^{6}$ Roel Verhaak, ${ }^{7}$ Shulin Li, ${ }^{8}$ and Amy B. Heimberger ${ }^{1}$
}

\begin{abstract}
'Department of Neurosurgery, The University of Texas MD Anderson Cancer Center, Houston, Texas, USA. ${ }^{2}$ Department of Microbiology \& Immunology, Dodson Interdisciplinary Immunotherapy Institute, Sylvester Comprehensive Cancer Center, University of Miami, Miami, Florida, USA. ${ }^{3}$ Departments of Biostatistics, ${ }^{4}$ Cancer Biology, and ${ }^{5}$ Neuropathology, The University of Texas MD Anderson Cancer Center, Houston, Texas, USA. ${ }^{6}$ School of Life Science and Technology, ShanghaiTech University, Shanghai, China. ${ }^{7}$ Jackson Laboratory of Genomic Medicine, Farmington, Connecticut, USA. ${ }^{8}$ Department of Pediatrics, The University of Texas MD Anderson Cancer Center, Houston, Texas, USA.
\end{abstract}

Glioblastoma is highly enriched with macrophages, and osteopontin (OPN) expression levels correlate with glioma grade and the degree of macrophage infiltration; thus, we studied whether OPN plays a crucial role in immune modulation. Quantitative PCR, immunoblotting, and ELISA were used to determine OPN expression. Knockdown of OPN was achieved using complementary siRNA, shRNA, and CRISPR/Cas9 techniques, followed by a series of in vitro functional migration and immunological assays. OPN gene-deficient mice were used to examine the roles of non-tumor-derived OPN on survival of mice harboring intracranial gliomas. Patients with mesenchymal glioblastoma multiforme (CBM) show high OPN expression, a negative survival prognosticator. OPN is a potent chemokine for macrophages, and its blockade significantly impaired the ability of glioma cells to recruit macrophages. Integrin $\alpha_{v} \beta_{5}$ (ITG $\left.\alpha v \beta 5\right)$ is highly expressed on glioblastoma-infiltrating macrophages and constitutes a major OPN receptor. OPN maintains the M2 macrophage gene signature and phenotype. Both tumor-derived and host-derived OPN were critical for glioma development. OPN deficiency in either innate immune or glioma cells resulted in a marked reduction in M2 macrophages and elevated T cell effector activity infiltrating the glioma. Furthermore, OPN deficiency in the glioma cells sensitized them to direct CD8 ${ }^{+} \mathrm{T}$ cell cytotoxicity. Systemic administration in mice of 4-1BB-OPN bispecific aptamers was efficacious, increasing median survival time by $68 \%(P<0.05)$. OPN is thus an important chemokine for recruiting macrophages to glioblastoma, mediates crosstalk between tumor cells and the innate immune system, and has the potential to be exploited as a therapeutic target.

\section{Introduction}

Glioblastoma multiforme (GBM) is one of the most malignant cancers, with a 5 -year survival rate of $5 \%$ despite aggressive treatment (1). To date, there has been significant research and clinical focus on the modulation of adaptive immune responses for cancer therapy. Unlike other malignancies, GBM is characterized by a paucity of T cell infiltration but robust macrophage infiltration. However, little is understood regarding the chemokines, pathways, and mechanisms that differentially regulate the influx of distinct immune cells, including macrophages, into CNS tumors such as GBM.

Osteopontin (OPN) is a glycophosphoprotein with an exposed arginine-glycine-aspartate-containing (RGD-containing) domain that is expressed both intra- and extracellularly by various cell types, including macrophages, epithelial cells, smooth muscle

Conflict of interest: The authors have declared that no conflict of interest exists License: Copyright 2019, American Society for Clinical Investigation.

Submitted: March 26, 2018; Accepted: October 4, 2018.

Reference information: J Clin Invest. 2019;129(1):137-149.

https://doi.org/10.1172/JCl121266. cells, osteoblasts, and cancer cells (2). The RGD site of OPN can bind to multiple integrins, such as $\alpha_{\mathrm{v}} \beta_{3}, \alpha_{\mathrm{v}} \beta_{5}, \alpha_{\mathrm{v}} \beta_{1}$, and $\alpha_{5} \beta_{1}$, and to certain variant forms of CD44 (3). OPN has a prominent role in glioma biology (4), and expression levels correlate with glioma grade $(5,6)$ and angiogenesis $(7)$. Elevated serum and cerebral spinal fluid OPN levels have been shown to correlate with poor prognosis in glioblastoma patients $(8,9)$. Mechanistically, OPN induces glioma cell migration and invasion (10-12), and OPN/ CD44 signaling in the glioma perivascular niche enhances cancer stem cell phenotypes and promotes aggressive tumor growth (13). Notably, selective OPN knockdown exerts antitumor activity in a human glioblastoma model (14).

OPN is also expressed in various immune cells and plays a role in initiating immune responses $(15,16)$. OPN is upregulated within glioblastoma-infiltrating neutrophils and macrophages, and is associated with infiltration of these cells within tumor specimens (17). Moreover, OPN promotes myeloid-derived suppressor cell expansion by activation of the STAT3 pathway (18) and suppresses antitumor immunity by promoting extramedullary myelopoiesis (19). Additionally, OPN upregulates COX-2 expression in tumor- 
A

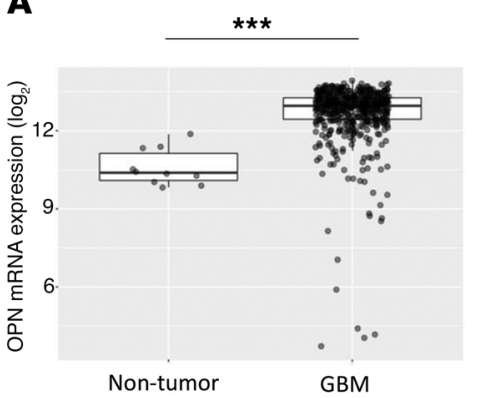

D

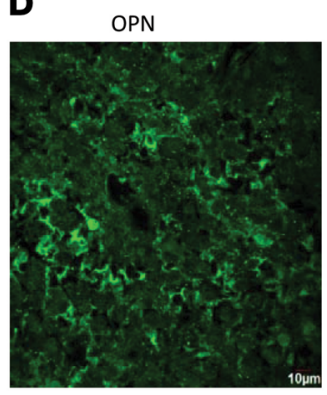

\section{B}

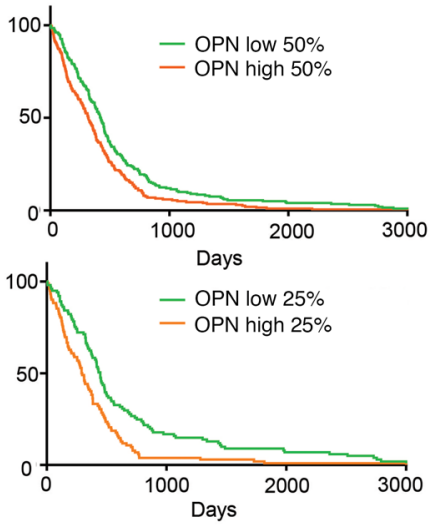

Days
C

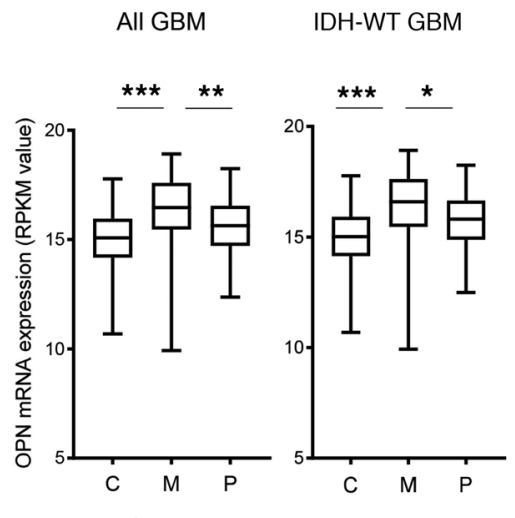

E

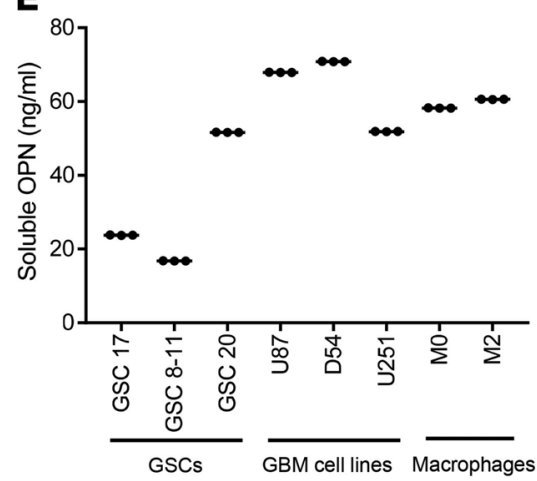

Figure 1. OPN expression is prognostic in human glioblastoma and associates with the mesenchymal subtype. (A) OPN expression is higher in GBM $(n=528)$ than in non-tumor brain tissue $(n=10, P=0.000000067)$ based on TCGA data. (B) Kaplan-Meier survival estimates of glioblastoma patients in relation to expression levels of OPN in the tumors based on TCGA data sets. Median survival time: OPN high-expressing (50\%) group, 318 days versus low-expressing (50\%) group, 422 days $(n=403)$; OPN high-expressing ( $25 \%)$ group, 447 days versus low-expressing (25\%) group, 296 days $(n=203)$. (C) OPN expression preferentially associates with the mesenchymal $(M ; n=51)$ versus the classical $(C ; n=59)$ or proneural (P; $n=46)$ CBM subtype (total $n=156$; M vs. C: $P=7 \times 10^{-6} ;$ M vs. $\left.P: P=0.0043\right)$. A similar preferential association was found in IDH-WT CBM $(n=139): \mathrm{M}(n=48)$ vs. C $(n=56): P=6 \times 10^{-6} ; \mathrm{M}$ vs. $P(n=35): P=0.0459$. In all GBMs (left), C: minimum ( $\min ) 10.68,25 \%$ percentile 14.17 , median $15.08,75 \%$ percentile 15.95 , maximum (max) $18.92 ;$ M: min 9.927, $25 \%$ percentile 15.46 , median $16.54,75 \%$ percentile 17.59 , max 18.92; P: min 12.37, $25 \%$ percentile 14.71 , median 15.64 , $75 \%$ percentile 16.54 , max 18.25 . In IDH-WT CBMs (right), C: $\min 10.68,25 \%$ percentile 14.13, median 15.02, 75\% percentile 15.92, max 17.77; M: min 9.927, 25\% percentile 15.45, median 16.59, 75\% percentile 17.62, $\max 18.92$; P: $\min 12.49,25 \%$ percentile 14.88, median 15.81, 75\% percentile 16.65, max 18.25. RPKM, reads per kilobase million. (D) Ex vivo human GBM immunofluorescently stained for expression of OPN (green) and glioma stem cells, denoted by SOX2 expression (red). Nuclei were stained with DAPI (blue) and the images merged, illustrating the prominent expression of OPN in the GBM tumor microenvironment. Original magnification, $\times 400$ (scale bars: $10 \mu \mathrm{m}$ ). (E) Human GSCs, glioma cell lines, and macrophages were analyzed for production of OPN at 48 hours by ELISA (cells were seeded at 0.5 $\times 10^{6} / \mathrm{ml}$ as a starting culture density). $P$ values were calculated using the 2 -tailed 2-sample $t$ test. Data indicate mean \pm SD and are representative of 3 independent experiments. ${ }^{* *} P<0.001,{ }^{* *} P<0.001,{ }^{*} P<0.05$.

associated macrophages, leading to enhanced angiogenesis and tumor growth via $\alpha_{9} \beta_{1}$ integrin (20). Cumulatively, these properties of OPN would make it a potentially applicable target for therapeutic manipulation within the context of GBM. Oligonucleotide-based aptamers represent an emerging platform technology for generating ligands with desired specificity that can be used to activate or antagonize immune mediators or receptors (21). OPN-specific aptamers have been developed and have shown therapeutic efficacy in inhibiting cancer growth $(22,23)$. Here we demonstrate that OPN is a chemokine that drives the infiltration of macrophages into GBM, plays a role in the immune-suppressive properties of the macrophage, and can be exploited as a therapeutic target.

\section{Results}

OPN is expressed in the glioblastoma microenvironment and is a negative prognosticator for survival. Bioinformatic analyses from
The Cancer Genome Atlas (TCGA) database showed that OPN expression is significantly elevated in GBM relative to nontumor brain tissues $\left(P=6.7 \times 10^{-8}\right)$ (Figure 1A). Furthermore, OPN expression negatively correlated with patient survival. When OPN expression was dichotomized based on a cutoff point of $50 \%$, higher levels of OPN expression were associated with poor prognosis $(P=0.001 ; n=403)$. The prognostic influence was even more apparent when the extremes of expression were analyzed based on a $25 \%$ cutoff point $(P=0.0002 ; n=203)$ (Figure 1B). OPN expression was enriched in the GBM mesenchymal subset, including when accounting for isocitrate dehydrogenase (IDH) WT status (Figure 1C); this is consistent with the previous observation that the mesenchymal subtype is enriched for inflammatory responses, but especially MO- and M2-polarized macrophages $(24,25)$. Because OPN has previously been shown to have a role in the activation and migration of macrophages in general 
A

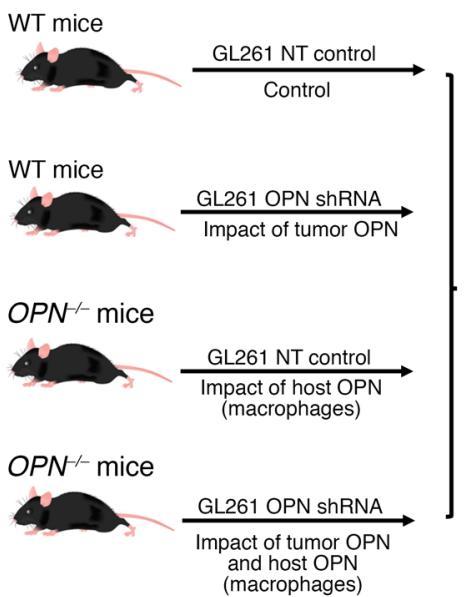

B

- GL261 NT control - WT mice

- GL261 OPN shRNA - WT mice

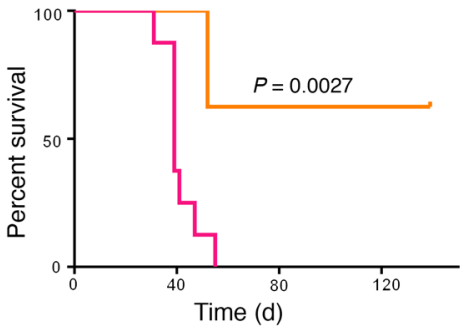

D

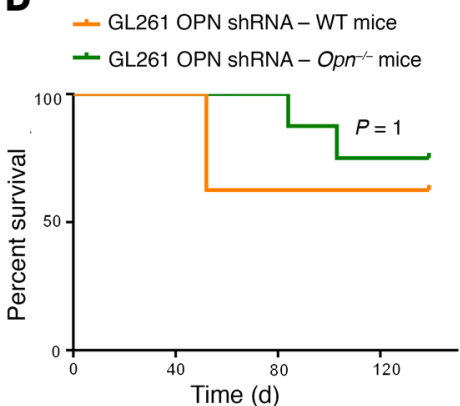

C

- GL261 NT control - WT mice

- GL261 NT control - Opn`^ mice

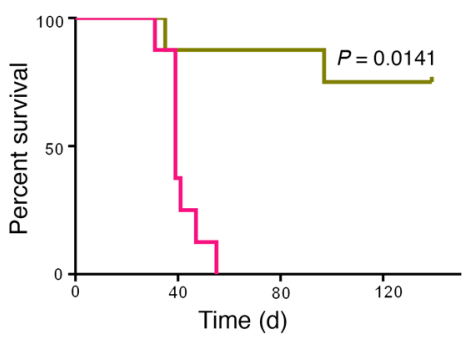

E

- GL261 NT control - WT mice

- GL261 OPN shRNA - WT mice

- GL261 NT control - Opn ${ }^{-}$mice

- GL261 OPN shRNA - Opn ${ }^{-}$mice

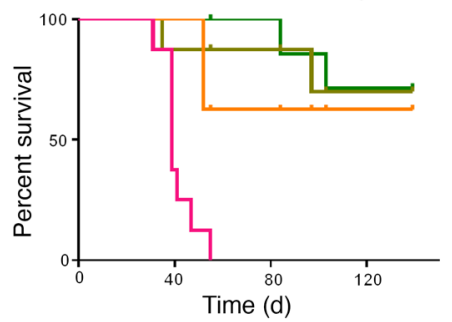

Figure 2. OPN expression in either the tumor or host impacts survival in a murine model of glioma. (A) Experimental schema to clarify the significance of OPN expression in glioma progression in various cellular contexts. Either WT or Opn ${ }^{-/}$background (KO) mice were intracranially implanted with GL261 cells that were transfected with either a non-targeting shRNA (NT control) or shRNA specific to OPN (OPN shRNA). (B) The median survival time was markedly increased in mice implanted with GL261 glioma cells transfected with OPN shRNA relative to GL261 controls (NT control) ( $n=9$ for GL261 OPN shRNA group, and $n=10$ for NT group; $P=0.0027$ ). (C) The median survival time of mice bearing intracranial GL261 tumors was also extended in the $O p n^{-/-}$background ( $n=10$ per group; $P=0.0141$ ). (D) There was no significant difference in survival if both glioma and host OPN expression was knocked out $(P=1)$. $P$ values were calculated based on the log-rank test with Bonferroni's correction. (E) Kaplan-Meier curves demonstrating survival of all groups for comparison. Data are representative of 2 independent experiments.

(26), using TCGA data set, we found that multiple macrophage markers, such as S100A9, CD68, CD14, and CD163, correlated well with OPN expression in tumor (Supplemental Figure 1A; supplemental material available online with this article; https://doi. org/10.1172/JCI121266DS1). In ex vivo human GBM specimens, dual immunofluorescence staining demonstrated OPN expression in association with $\mathrm{SOX}^{+}$GBM tumor cells (Figure 1D and Supplemental Figure 1B). Based on ELISA quantification of OPN released into supernatants, both glioblastoma stem cells (GSCs), with an average production of $25,697 \mathrm{pg} / \mathrm{ml}$ (range, 16,785$51,644, n=3$ ), and glioma cell lines, with an average production of $63,543 \mathrm{pg} / \mathrm{ml}$ (range, $51,856-70,850, n=3$ ), are cellular sources of elaborated OPN. Human MO and M2 macrophages also secrete OPN in the range of 58,248-60,620 pg/ml (Figure 1E). Cumulatively, these data indicate that there is a dual source of elaborated OPN: the tumor cells and the macrophages.

OPN expression in tumor cells and non-tumor cells influences survival equally in GL261 glioma-bearing mice. To ascertain whether the expression of OPN in tumor cells versus non-tumor cells has a role in survival outcome, we implanted OPN shRNA-transfected and non-targeting (NT) shRNA control-transfected GL261 glioma cells (which produced $105.4 \mathrm{pg} / \mathrm{ml}$ and $1536.2 \mathrm{pg} / \mathrm{ml}$ OPN, respectively) intracranially in both WT C57BL/6 mice and mice on an OPNdeficient background $\left(\mathrm{Opn}^{--}\right)$(Figure 2A). Transfection of the GL261 cells with the OPN shRNA did not alter cell morphology, proliferation, or viability (Supplemental Figure 2). The median survival time of mice harboring intracerebral GL261 on the WT background was 39 days, whereas the median survival could not be ascertained in the GL261 OPN shRNA mice because 6 of 9 mice were long-term survivors $(P=0.0027)$, which suggests that tumor-derived OPN plays an important role in glioma progression (Figure 2B). Next, we implanted GL261 NT glioma cells intracranially in either WT or $\mathrm{Opn}^{-1-}$ syngeneic mice to examine the nontumor/host OPN contribution to glioma progression. The median survival time of GL261 glioma cells implanted in mice on the WT background was 40 days, whereas the median survival could not be ascertained in mice implanted with GL261 gliomas on the $\mathrm{Opn}^{-/-}$ background ( $P=0.0047$, Figure $2 \mathrm{C})$. We then explored whether there was a further survival benefit when OPN was ablated in both the glioma cells and in the host. The median survival times could not be ascertained for either WT or $\mathrm{Opn}^{--}$mice implanted with GL261 shOPN glioma cells, given that most of the mice were long-term survivors. A trend was observed such that more GL261 shOPN-implanted mice survived in the $\mathrm{Opn}^{-1-}$ than the WT group, but this was not statistically significant $(P=0.3676$, Figure 2D). Cumulatively, these survival data demonstrated that both tumorand host-derived OPN are key contributors to glioma progression.

OPN deficiency enhances $T$ cell effector activity and reduces glioma macrophage infiltration. Because OPN has documented immune-modulatory effects, to elucidate the immune mechanisms responsible for the prolonged survival of GL261 gliomabearing mice with OPN diminishment, we investigated immune 
cell composition and functional change. The 4 groups of gliomabearing mice ( $n=8$ per group) - NT shRNA GL261-implanted WT mice, OPN shRNA GL261-implanted WT mice, NT shRNA GL261-implanted $\mathrm{Opn}^{-/}$mice, and OPN shRNA GL261-implanted $\mathrm{Opn}^{-/-}$mice - were euthanized on day 14 to isolate immune cells from the brain tumor, spleen, and blood. There was a statistically significant decrease in the number of M2 macrophages $\left(\mathrm{CD} 206^{+} \mathrm{CD}_{\left.11 \mathrm{~b}^{+}\right)}\right.$ residing in the spleen regardless of whether the OPN deficiency was present in the glioma (GL261 OPN shRNA-WT), the background (GL261 NT-KO), or both (GL261 OPN shRNA-KO) when compared with the WT mice implanted with NT shRNA GL261 glioma (Figure $3 \mathrm{~A})$. Similarly, the number of macrophages $\left(\mathrm{F} 4 / 80^{+} \mathrm{CD} 11 \mathrm{~b}^{+}\right)$infiltrating the brain tumor was significantly reduced in these groups (Figure 3A). Histological staining of Iba-1 validated the flow cytometry findings, with macrophage glioma infiltration markedly diminished in the OPN-deficient mice (Figure 3B). The frequency of both $\mathrm{CD}^{+}$and $\mathrm{CD}^{+}$IFN- $\gamma$-producing $\mathrm{T}$ cells was increased in spleen, blood, and brain tumors in the OPN-deficient glioma mice (Figure 3C). Likewise, there was an increased frequency of TNF- $\alpha-$ producing $\mathrm{CD}^{+}$ and $\mathrm{CD}^{+} \mathrm{T}$ cells in the OPN-deficient models (Supplemental Figure $3 \mathrm{~A})$. An increase in IL-2-producing $\mathrm{CD}^{+}$and $\mathrm{CD} 8^{+} \mathrm{T}$ cells was found in the glioma (Figure 3D) but not in the blood and spleen (Supplemental Figure 3B). There was a notable reduction in the frequency of immune-suppressive FoxP3 ${ }^{+}$Tregs in the blood of OPN-deficient models (Figure $3 \mathrm{E}$ ), but there was no reduction in the frequency of Tregs in the spleen or brain tumor (Supplemental Figure 3C). To exclude the possibility that significant changes in the peripheral and intratumoral immune cell subsets result from decreases in other unexamined subpopulations of cells rather than expansion of the examined IFN- $\gamma^{+}$and IL-2 $2^{+} \mathrm{T}$ cell subsets, we assessed absolute cell counts, with similar findings (Supplemental Figure 4). These data indicate that the percent changes in the $\mathrm{T}$ cell subsets were mainly due to their expansion. This also applied to the decrease in CD206 ${ }^{+}$ spleen macrophages, $\mathrm{F} 4 / 80^{+}$tumor macrophages, and peripheral $\mathrm{FoxP}^{+}$Tregs (Supplemental Figure 4, A and D). Cumulatively, these data indicate that the reduction in M2 macrophage infiltration and elevation of $\mathrm{T}$ cell effector activity are responsible for the prolonged survival of glioma mice with OPN deficiency, and that OPN plays a pivotal role in antagonizing $\mathrm{T}$ cell effector function.

OPN deficiency renders glioma cells more sensitive to $T$ cell killing. We have previously shown that GSCs are suppressive of both innate and adaptive immunity $(27,28)$. To investigate the possible OPN downstream immune mediators, such as cytokines and chemokines, we quantified expression levels in supernatants from 5 pairs of OPN-knockdown GSCs. Based on the cytokine and chemokine screening results, there was minimal to no change in soluble factors participating in immune surveillance or suppression, such as CCL2, CCL5, IL-2, IL-6, IL-10, IL-12, IFN- $\gamma$, TNF- $\alpha$, and TGF- $\beta$, etc. (Supplemental Table 1). Functional immune assays also demonstrated that OPN deficiency in the GSCs did not alter their ability to suppress $\mathrm{T}$ cell proliferation, induce $\mathrm{T}$ cell apoptosis, or generate FoxP3 ${ }^{+}$Tregs (Supplemental Figure $5)$. Because there were no alterations in soluble factors found to be responsible for enhancement of in vivo immune clearance, we next determined whether there was increased $\mathrm{CD}^{+} \mathrm{T}$ cell killing activity against OPN-deficient GL261 tumor cells based on direct cell-cell contact. At T cell-to-glioma cell ratios of 5:1 and 10:1, there was a significant increase in murine $\mathrm{CD}^{+} \mathrm{T}$ cell cytotoxicity against OPN-deficient GL261 cells (Supplemental Figure 6A). Furthermore, the $\mathrm{T}$ cells had more robust production of an effector response, such as IFN- $\gamma$, when cocultured with OPN-deficient GL261 cells (Supplemental Figure 6B). These data suggest that OPN deficiency in tumor cells can sensitize them to $\mathrm{CD}^{+} \mathrm{T}$ cell killing in a cell-cell contact manner.

OPN attracts tumor-supportive macrophage migration to the glioma. Recombinant OPN and conditioned medium from human GSCs attracted MO and M2 macrophages (Figure 4A), which predominate in the glioblastoma microenvironment (29). To further address whether the OPN-mediated attractant effect is dose dependent, we examined the impact of different dilutions of OPN protein on MO macrophage migration and found a dose-dependent effect (Figure 4B). OPN could attract M1 macrophages, albeit with a reduced capacity (Supplemental Figure 7A). Preincubation of GSC-conditioned medium with an anti-OPN antibody attenuated the OPN-promoting effect of macrophage migration (Figure 4C). Furthermore, OPN KO in GSCs by CRISPR/Cas9 (Supplemental Figure 8) also significantly reduced the chemoattractant effect (Figure 4D), demonstrating the importance of OPN as a macrophageattracting factor in GBM.

OPN mediates tumor-supportive macrophage migration to the glioma through $\alpha_{v} \beta_{5}$ integrin signaling. To clarify the mechanism of OPN-mediated recruitment of macrophages and to ascertain potential binding partners, we analyzed the expression profile of integrin family members on glioblastoma-infiltrating macrophages (GIMs) relative to matched sets of PBMCs and healthy donor monocytes from patients. Integrin $\beta_{5}$ (ITG $\beta 5$ ) and integrin $\alpha_{v}$ (ITG $\alpha$ ) were the most-upregulated integrin family members (Figure 5A), and these integrins form an $\alpha_{v} \beta_{5}$ heterodimer. The expression of integrin $\alpha_{v} \beta_{5}$ on GIMs was validated with flow cytometry analysis of ex vivo human GBMs (Figure 5B). Furthermore, $\alpha_{\mathrm{v}} \beta_{5}$ was confirmed to be expressed on MO and M2 macrophages (Figure $5 \mathrm{C}$ ), which is the functional phenotype assumed by the GIMs (29). When these MO and M2 macrophages were preincubated with $\alpha_{\mathrm{v}} \beta_{5}$ blocking antibody, they failed to respond to exogenous OPN $(10 \mathrm{ng} / \mathrm{ml})$ for their migration (Figure 5D), and parallel blockade of $\alpha_{\mathrm{v}} \beta_{5}$ on M0 and M2 macrophages completely abolished their migration to GSC conditioned medium (Figure 5E). The $\alpha_{v} \beta_{5}$ ligand is specific to the chemotactic properties of OPN, since $\alpha_{v} \beta_{5}$ blockade did not inhibit the macrophage chemotactic response to CCL2, another well-known macrophage chemokine (Supplemental Figure 7B). Cumulatively, these data indicate that OPN in the GBM microenvironment can induce the attraction of macrophages through integrin $\alpha_{\mathrm{v}} \beta_{5}$ signaling.

OPN and integrin $\alpha_{1} \beta_{5}$ are upregulated in GIMs. Because the murine glioma model in $\mathrm{Opn}^{-/-}$mice suggested that OPN expression in non-tumorigenic cells plays a critical role in glioma progression and because OPN is known to be upregulated during macrophage differentiation (30) and mediates survival, phagocytosis, and inflammatory responses $(31,32)$ in macrophages, we next sought to ascertain its potential role within GIMs, which assume a functional phenotype in the continuum of MO to M2 (29). Thus, we conducted gene array profiling of human CD14 monocytes isolated ex vivo from glioblastoma, which are the precursors to macrophages and are the source of GIMs. Among 25,000 genes that are upregulated in $\mathrm{CD} 14^{+}$GIMs relative to their 
A

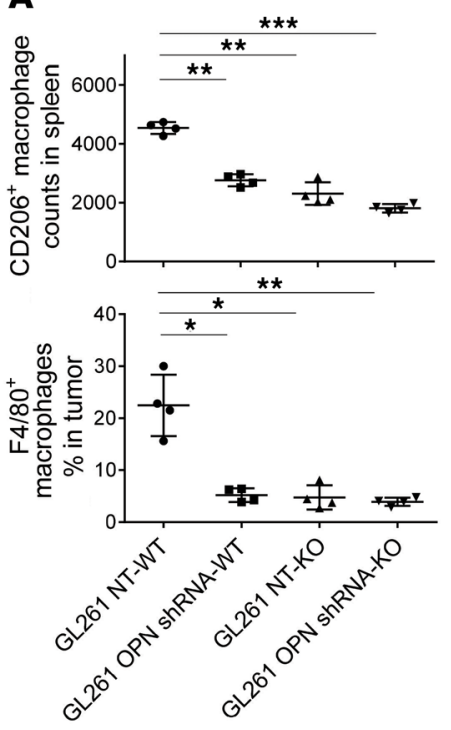

B GL261 NT-WT GL261 OPN shRNA-WT

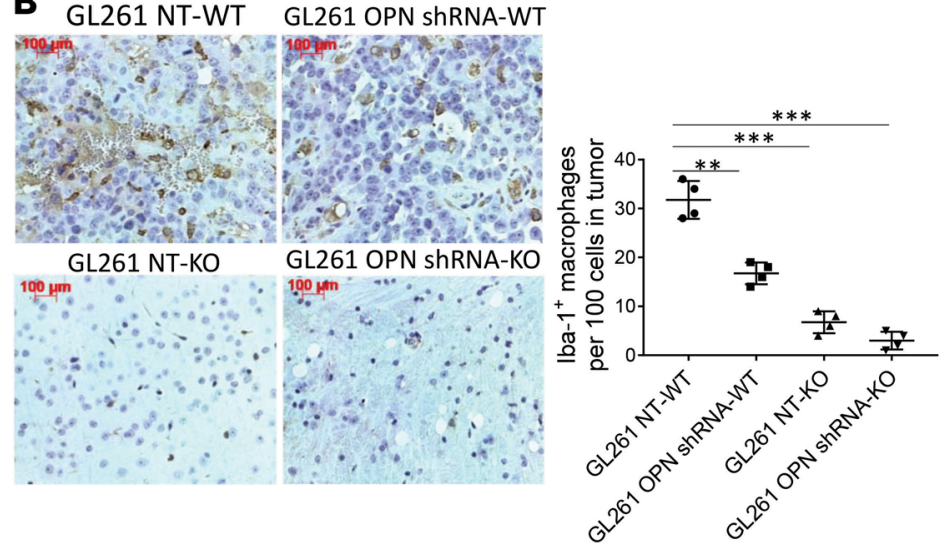

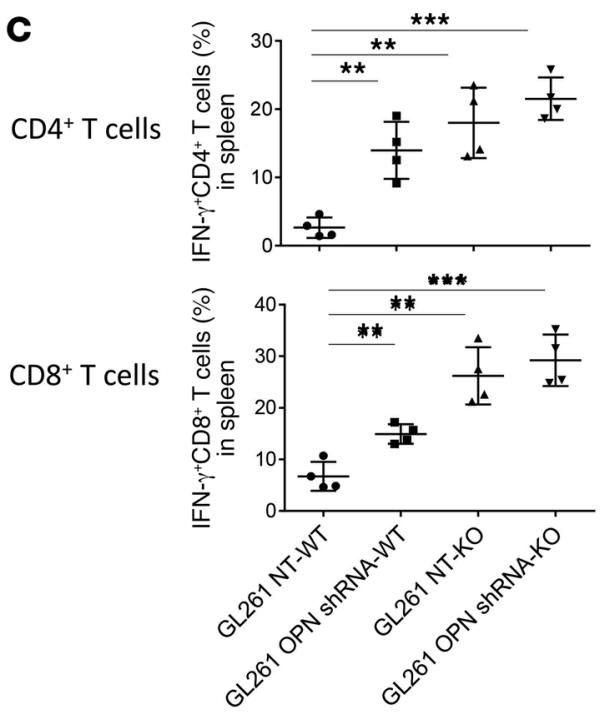
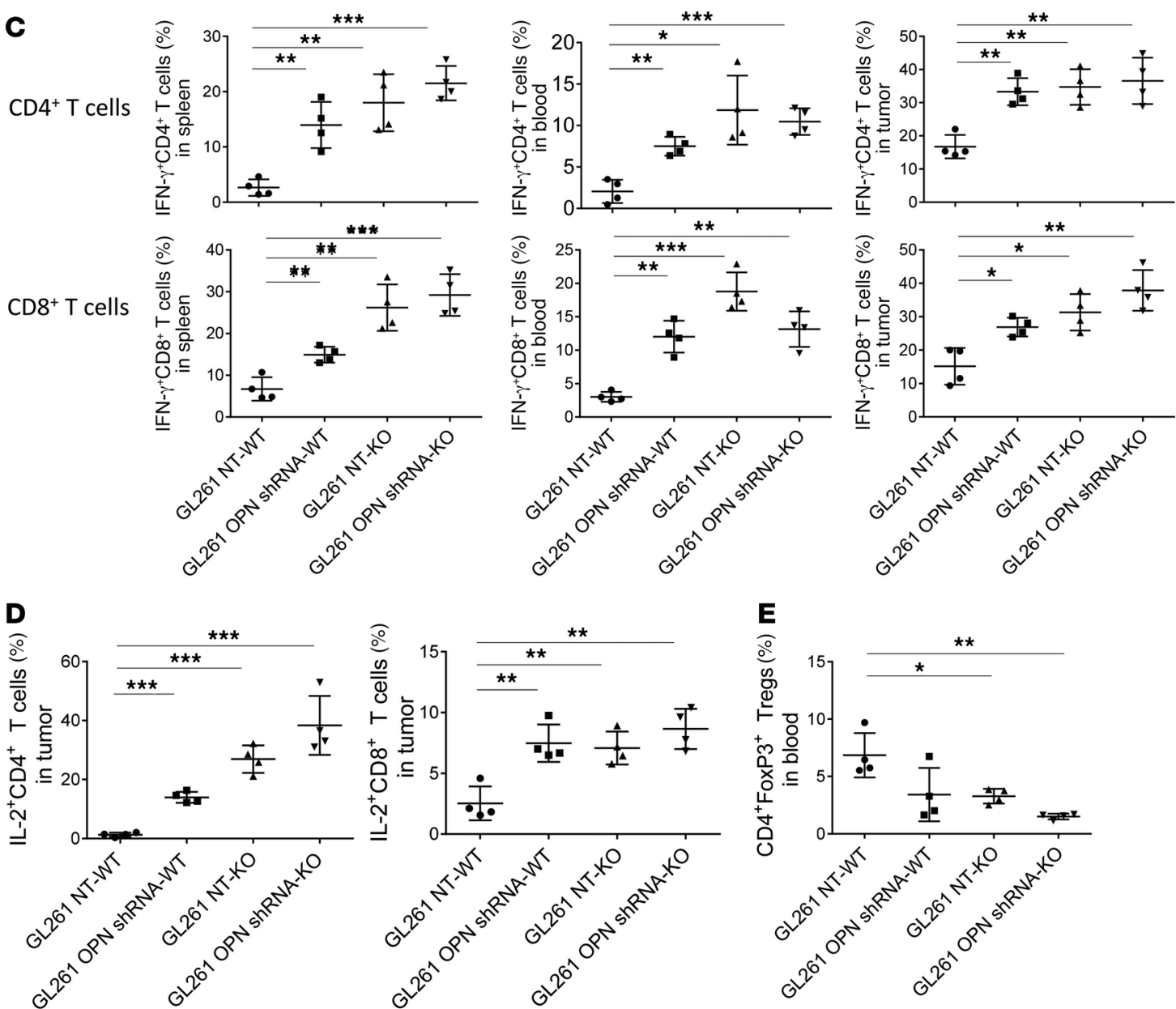

Figure 3. Reduction of macrophage infiltration and enhanced T cell effector activity in the tumor microenvironment is due to OPN loss. Either WT or Opn ${ }^{-1-}$ background mice were intracranially implanted with GL261 cells that were transfected with an NT control shRNA or shRNA specific to OPN (OPN shRNA) (50,000 cells per mouse, 8 mice/group, 4 groups). All mice were euthanized on day 14, and the tumor brain tissue, spleen, and blood were collected for ex vivo flow cytometry and immunohistochemical staining. (A) Percentages of CD206+CD11b+ macrophages in the spleen and F4/80+CD11 b+ macrophages in the brain tumor were diminished when either OPN in the tumor or in the host background was knocked out. (B) Representative immunohistochemistry showing the frequency of Iba-1 macrophages in the tumor; the graph summarizes the quantitative results. Original magnification, $\times 100$ (scale bars: $100 \mu \mathrm{m}$ ). (C) Percentage of IFN- $\gamma^{+}$CD4 ${ }^{+}{ }^{+}$cells and IFN- $\gamma^{+}$CD8 ${ }^{+}$effector T cells in the spleen (left), PBMCs (middle), and tumor (right). (D) Percentage of IL-2+CD4+ $\mathrm{T}$ cells (left) and IL-2+CD8 ${ }^{+}$effector T cells (right) in the tumor. (E) Percentage of CD4+FoxP3 ${ }^{+}$Tregs in PBMCs. Data represent mean \pm SD of 4 different animals in 1 experiment. $P$ values were calculated based on 2-tailed 2-sample $t$ test with Bonferroni's correction. ${ }^{*} P<0.05,{ }^{* *} P<0.01,{ }^{* * *} P<0.001$. 
A

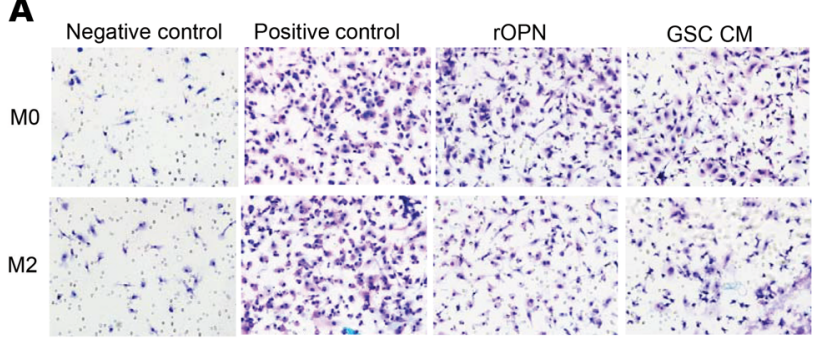

B

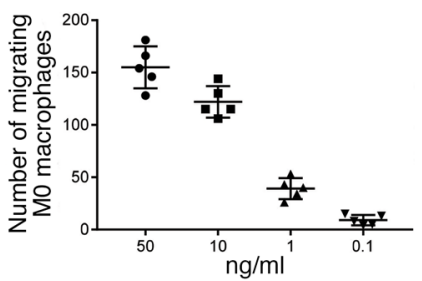

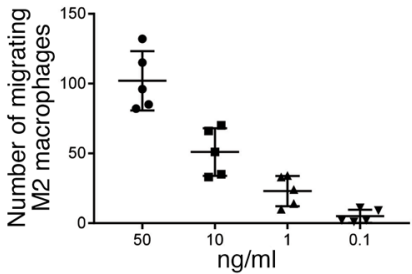

GSC CM

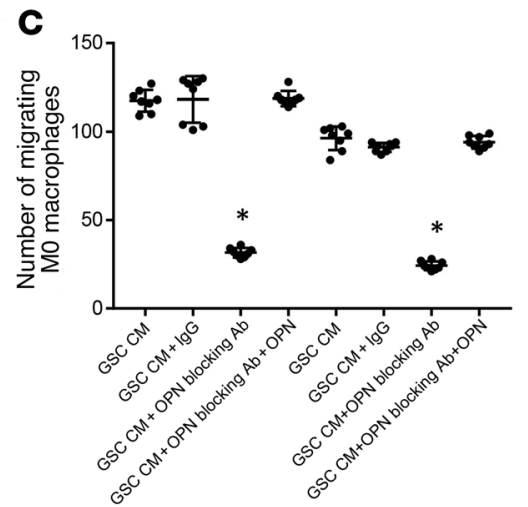

D
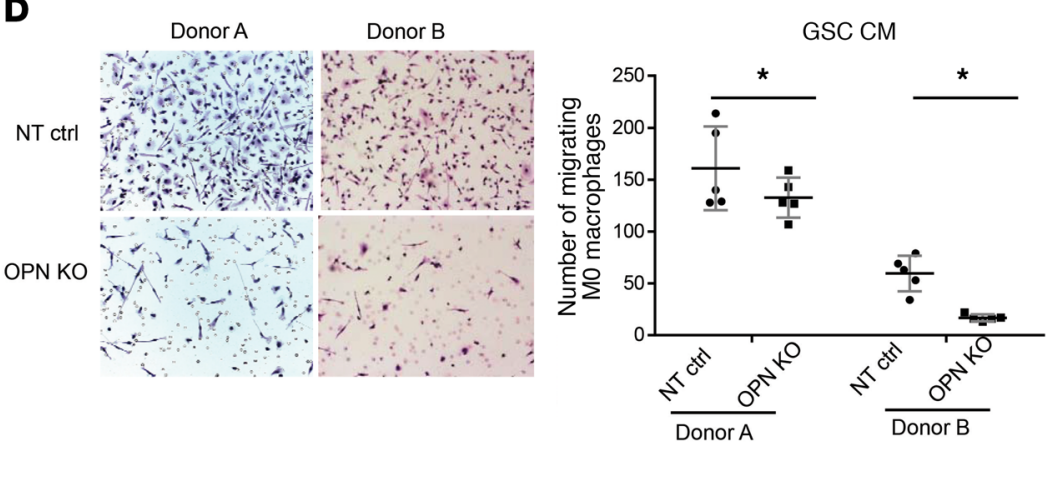

Figure 4. OPN is chemotactic for macrophages. (A) Transwell migration assays of human MO- and M2-skewed macrophages exposed to serum-free medium (negative control), 10\% FBS (positive control), recombinant OPN (rOPN, $50 \mathrm{ng} / \mathrm{ml}$ ), and conditioned medium (CM) from GSCs at 48 hours. Original magnification, $\times 100$. (B) Sequential dilution of CSC CM demonstrating a dose response of OPN for macrophage migration. Data indicate mean \pm SD and are representative of 3 independent experiments. (C) Supernatants from GSCs were treated with $10 \mu \mathrm{g} / \mathrm{ml}$ of OPN-neutralizing antibody or the isotype lgG control and then applied to the lower chamber in the presence or absence of $10 \mathrm{ng} / \mathrm{ml} \mathrm{OPN}$ for attracting MO and M2 macrophages. Data are shown as mean \pm SD and are representative of 3 independent experiments. (D) Supernatants from OPN sgRNA/CRISPR (OPN KO) and NT scramble GSC cell lines (NT ctrl) were used to induce migration of M0 macrophages. Original magnification, $\times 100$. Data are shown as mean \pm SD and are representative of 2 independent experiments. Similar results were obtained using M2 macrophages (data not shown). $P$ values were calculated using the 2-tailed 2-sample $t$ test. ${ }^{*} P<0.05$.

matched $\mathrm{CD} 14^{+}$peripheral monocytes, OPN was the most significantly upregulated candidate (Figure 6A). OPN quantitative PCR of GIMs and their matched peripheral monocytes ( $n=11$ pairs) validated this finding (Figure 6B). To further clarify the cellular sources of OPN within human glioblastoma, we dually stained tumors for OPN and macrophages (Iba-1). Frequently, there was colocalization of OPN and Iba-1 throughout the glioblastoma, and in many instances, OPN could be found within the macrophages (Figure 6C). To evaluate whether these same observations hold in murine models, C57BL/6J mice harboring syngeneic GL261 intracerebral gliomas were euthanized, and their gliomas were stained with an anti-OPN and an anti-Iba-1 antibodies. As in human gliomas, the expression of OPN was heterogeneous throughout the tumor and also found to co-associate with $\mathrm{Iba}-1^{+}$macrophages (Supplemental Figure 9A).

Finally, to confirm that the GIMs express the integrin heterodimer $\alpha_{v} \beta_{5}$, these cells were isolated to evaluate expression of ITG $\alpha$ v and ITG $\beta 5$. Although ITG $\alpha$ v is expressed on normal donor monocytes $(n=3)$, in GBM patients' monocytes $(n=3)$, and their matched GIMs $(n=3)$, appreciable ITG $\beta 5$ levels were only found to be expressed on the GIMs (Figure 6D; uncut gels are presented in the supplemental material). Thus, we propose that GSCs and or GBM tumor cells secrete OPN, which is a chemokine that recruits MO and M2 macrophages via ITGav 35 ; these MO/M2 GIMs then play a role in tumor promotion and immune suppression and also secrete OPN, which further enhances the recruitment of $\mathrm{MO}$ and M2 macrophages (Figure 6E).
OPN does not induce macrophage skewing. To ascertain whether OPN plays a role in macrophage polarization, we treated MO macrophage precursors ( $n=3$ donors) with $10 \mathrm{ng} / \mathrm{ml}$ of OPN, a nonspecific siRNA control, and an OPN siRNA for 48 hours, and then performed FACS to detect the expression of M1 and M2 markers. There were no changes in the MFI of these markers (Supplemental Figure 10A). To determine whether OPN might induce the upregulation of immune checkpoint ligands such as $\mathrm{B} 7-\mathrm{H} 1,-\mathrm{H} 2,-\mathrm{H} 3$, or $-\mathrm{H} 4$, we obtained PBMCs from normal healthy donors and isolated CD14 monocytes $(n=3)$. OPN $(10,50$, and $100 \mathrm{ng} / \mathrm{ml})$ was coincubated with these cells for 48 hours, but there were also no changes in the MFI of the immune checkpoint ligand expression (Supplemental Figure 10B). These data indicate that although OPN may attract tumor-supportive macrophages, it is not a dominant mechanistic influence on macrophage polarization.

$O P N$ is required for M2 macrophage gene signature maintenance. Because the majority of GIMs assume a phenotype along the MO-to-M2 continuum (29), we next assessed whether OPN was preferentially expressed in distinctly polarized macrophages. Normal donor $\mathrm{CD}_{14}{ }^{+}$monocytes were isolated and polarized to the various macrophage phenotypes. M0, M1, and M2 polarization was verified based on marker expression of IL-2, CD163, CD204, TGF- $\beta$, and VEGF, as we have previously described (29). In culture, the M2 macrophage took on a more adhesive phenotype, whereas the M1 cells clustered in clumps. The highest levels of OPN, based on MFI, were found in the M2 macrophage, followed by the MO macrophage (4 days of GM-CSF culture). OPN expression was 
A

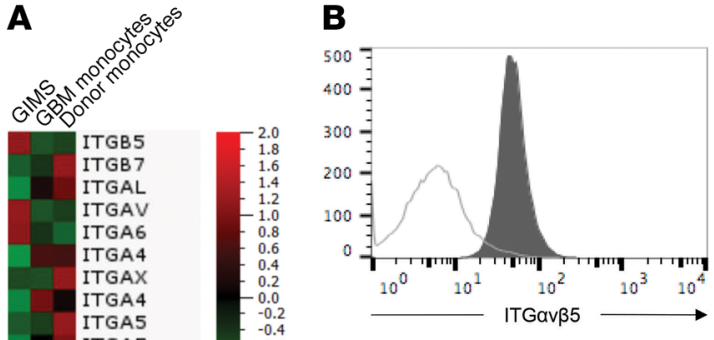

ITGAE

ITGAM

ITGB 1 BP 1

ITGB $1 B P 1$

ITGB3BP

ITGB 3BP

ITGB 2

ITGB $1 B P 2$

ITGB1BP3

ITGB 4

ITGAD
ITGA7

ITGA7

ITGA2 2

ITGB 6

ITGB 4

ITGA1

ITGB3

ITGA 11

ITGAD

ITGA3

ITGA2B

ITGA 10

ITGB 8

ITGB 1

ITGA10

C
D
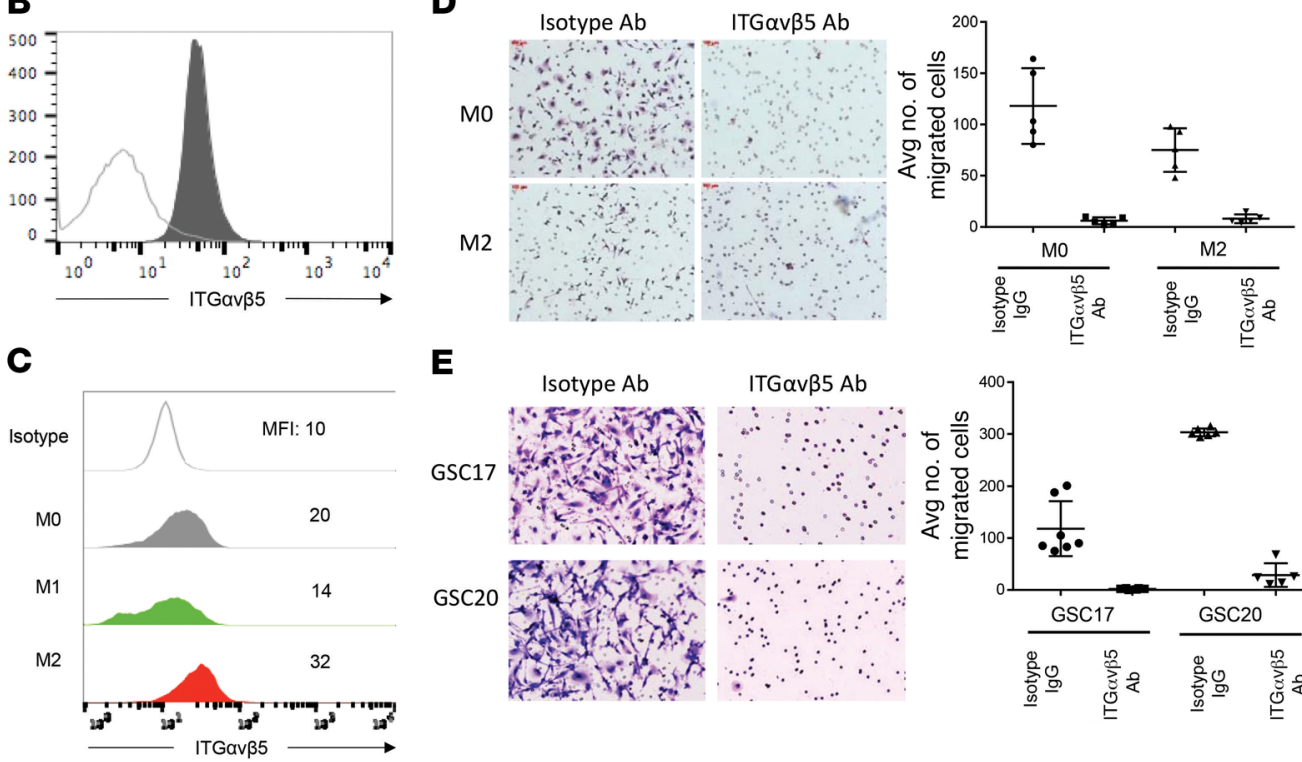

E

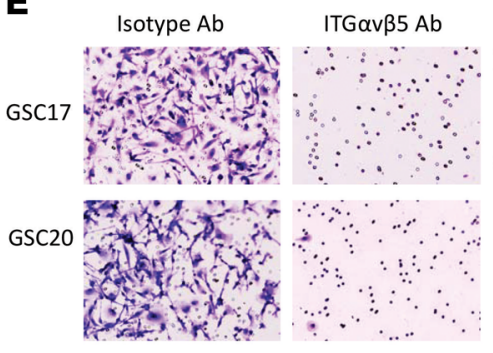

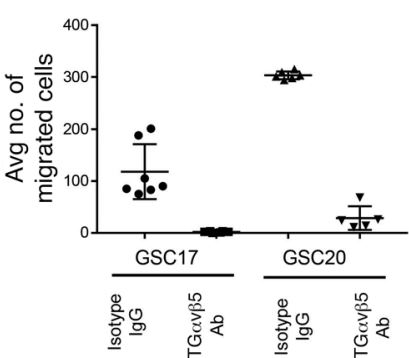

Figure 5. GSC OPN-mediated migration of GIMs requires ITG $\alpha \mathbf{\beta} \beta \mathbf{5 5}$. (A) An integrin expression panel was used to screen the preferential integrin expression of GIMs relative to the same GBM patient's peripheral blood monocytes and those of a normal non-tumor-bearing donor. (B) Flow cytometry verifying the expression of ITG $\alpha \mathrm{V} \beta 5$ on the surface of GIMs. Representative FACS data are shown from 3 independent experiments. (C) Representative flow cytometry histogram demonstrating that MO- and M2-skewed macrophages have enhanced expression of ITG $\alpha$ v $\beta 5$. MFI is shown. Representative FACS data from 3 independent experiments are shown. (D) Transwell migration assay of MO and M2 macrophages pretreated with ITG $\alpha \mathrm{V} \beta 5$-blocking antibody or its matched isotype antibody $(10 \mu \mathrm{g} / \mathrm{ml})$ and then exposed to $10 \mathrm{ng} / \mathrm{ml}$ OPN. Original magnification, $\times 100$ (scale bars: $100 \mu \mathrm{m})$. Avg, average. (E) Transwell migration assay of MO macrophages pretreated with ITG $\alpha v \beta 5$-blocking antibody or its matched isotype antibody $(10 \mu \mathrm{g} / \mathrm{ml})$ and then exposed to GSC conditioned medium. Similar results were obtained with M2 macrophages. Original magnification, $\times 100$. Data indicate mean \pm SD and are representative of 3 independent experiments.

lowest in M1 macrophages (Figure 7A). We investigated the role of OPN expression in M2 macrophages by knocking down OPN with its specific siRNAs and analyzing the alteration of M1- and M2associated signature genes. More specifically, M2 macrophages were polarized from naive $\mathrm{CD} 14^{+}$monocytes $(n=6)$, transfected with pooled OPN siRNAs on day 6 , and then harvested for RNA extraction 2 days later. OPN knockdown was verified with quantitative PCR (Figure 7B). When NanoString technology-based M1/M2 gene expression was performed, 8 M2-associated genes were significantly reduced in M2 macrophages, including PPAR $\gamma$, AdORA3, IRF8, HIF-2 $\alpha$, P2R $\gamma 5$, IRF5, TGF- $\beta 1$, and NF-kB $(P<$ 0.05 ), whereas no genes were upregulated (Figure $7 C$ ). Furthermore, phagocytic function was markedly enhanced upon OPN siRNA transfection (Figure 7D), but the presence of this functional feature is insufficient to support a claim that it represents a conversion to the M1 phenotype, since both M1 and M2 phenotypes are capable of phagocytosis (33).

4-1BB-OPN aptamer induces a therapeutic response in GL261 intracerebral models. Because of the profound survival advantage conferred when OPN is knocked out in either the glioma cell or host, we next screened several therapeutic strategies for potential efficacy against intracranial gliomas. A previously described OPN aptamer (OPN-R3) $(22,23)$ was capable of blocking MO and M2 macrophage migration (Figure $8 \mathrm{~A}$ ) but lacked in vivo therapeutic activity (Supplemental Figure 11A). Furthermore, ex vivo analysis demonstrated no difference between Iba- $1^{+}$macrophage infiltration in OPN-R3-treated tumors and controls (data not shown), nor was there a reduction in OPN gene expression (Supplemental Figure 9B). Additionally, an OPN-blocking monoclonal antibody did not exert therapeutic activity (Supplemental Figure 11A). Because OPN expression was so robust within the glioma, we devised an alternative therapeutic strategy of exploiting this expression as a way to trigger $\mathrm{CD} 8^{+} \mathrm{T}$ cell activation within the tumor. A 4-1BB aptamer can engage the costimulatory receptors on $\mathrm{CD} 8^{+} \mathrm{T}$ cells and promote the survival and expansion of $\mathrm{CD} 8^{+} \mathrm{T}$ cells (34). When the immune-stimulatory aptamer is conjugated to an OPNtargeting aptamer, a costimulatory response could theoretically be triggered within the tumor microenvironment that expresses OPN (21). To test the therapeutic potential of the $4-1 \mathrm{BB}-\mathrm{OPN}$ aptamer conjugate, we treated mice with established GL261 tumors i.v. with the aptamers either individually, mixed together, or in a conjugated format. Only the 4-1BB-OPN aptamer induced long-term durable therapeutic responses (Figure 8B). To ascertain whether there was preferential homing of the 4-1-BB-OPN aptamer to the glioma within the CNS, a complementary RNA probe to $4-1 \mathrm{BB}$ was used for in situ localization. Within the glioma, only the 4-1BB-OPN aptamer was detected, whereas a control 4-1BBprostate-specific membrane antigen (4-1BB-PSMA) aptamer that 
A

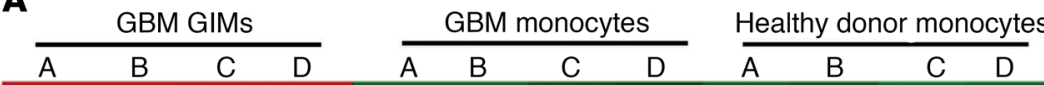

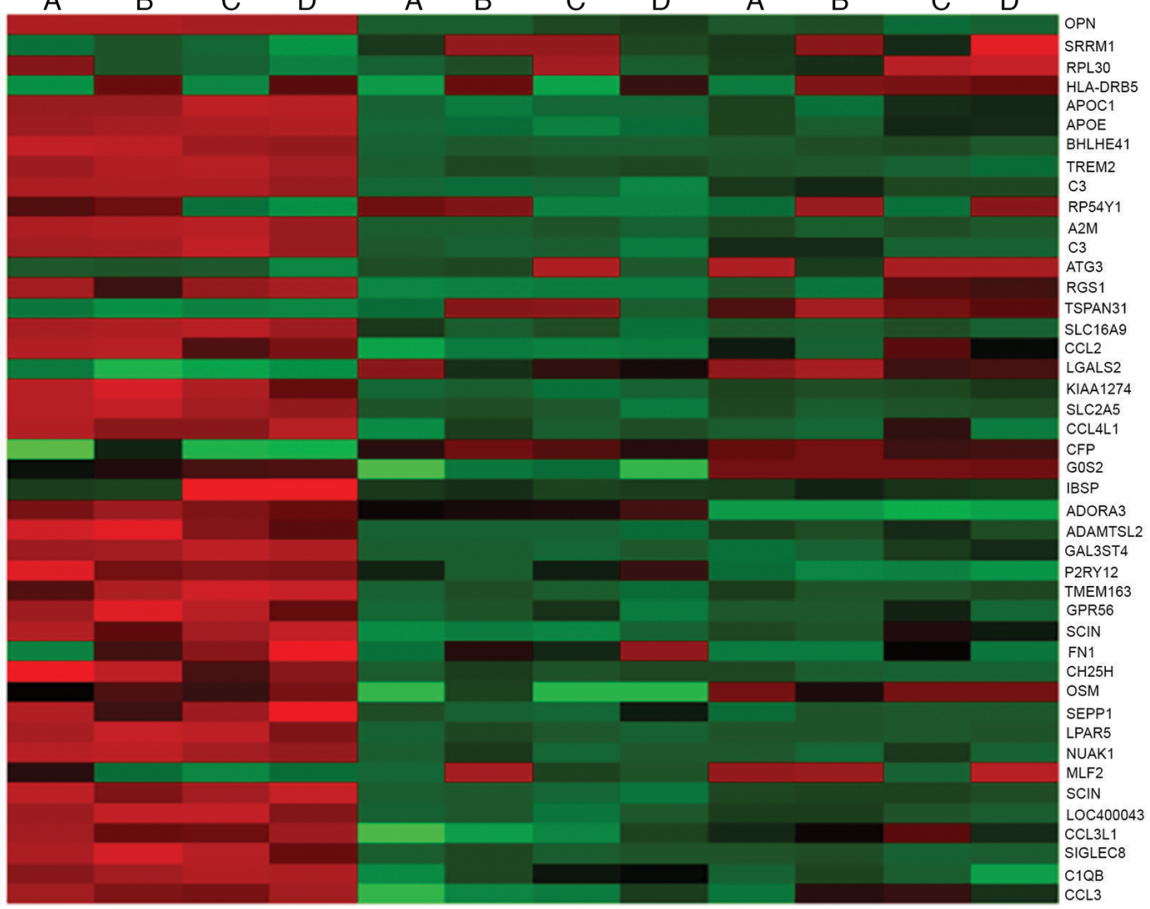

Figure 6. OPN is expressed in GIMs. (A) Heatmap demonstrating the preferential upregulation of genes in GIMs relative to matched patient peripheral blood monocytes. (B) In newly diagnosed GBM patients, quantitative PCR was used to evaluate the relative expression of OPN in matched specimens of CBM CD14+ ${ }^{+}$IIMs and monocytes $(n=10)$. Ct values were normalized to $\mathrm{GAPDH}$. Data are presented as mean \pm SEM and are representative of 3 independent experiments. (C) Immunofluorescence staining of OPN (green) and macrophages (lba-1, red) in an ex vivo GBM, including a merged (nuclei, DAPI, blue) and enlarged example. Original magnification, $\times 400$ (scale bars: $10 \mu \mathrm{m}$ ) and $\times 600$ (scale bars: 2 $\mu \mathrm{m})$. Graph: Iba- $1^{+} \mathrm{OPN}^{+}$macrophages in the GBM tissue. Data are shown as mean \pm SD and represent 3 independent experiments. (D) Western blots demonstrating that ITG $\alpha v \beta 5$ is preferentially expressed in the GIMs. (E) Unifying schema for the role of OPN in macrophages within the GBM tumor microenvironment. CBM tumor cells including CSCs elaborate OPN, which acts as a chemokine for $\mathrm{MO}$ and $\mathrm{M} 2 \mathrm{ITG} \alpha \mathrm{V} \beta 5$-expressing cells. GIMs are mostly in a phenotypic continuum of MO to M2 and express ITGav $\beta 5$. The GIMs also express OPN, which further amplifies the recruitment of additional $\mathrm{MO}$ - and $\mathrm{M} 2$-polarized cells into the tumor microenvironment.

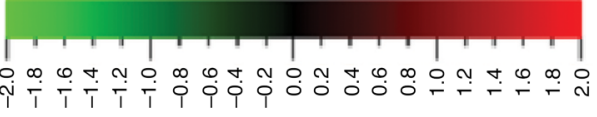

B

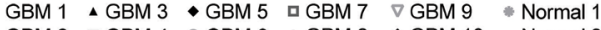

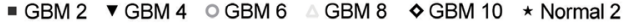

\section{音}
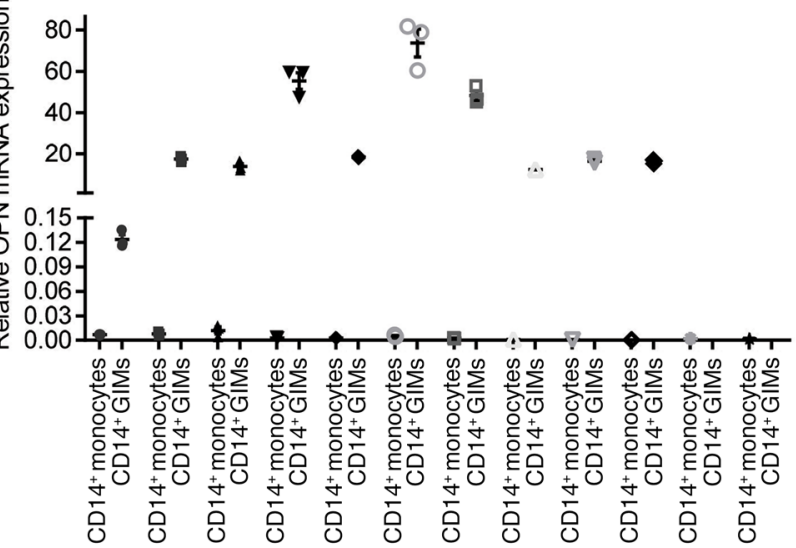

C

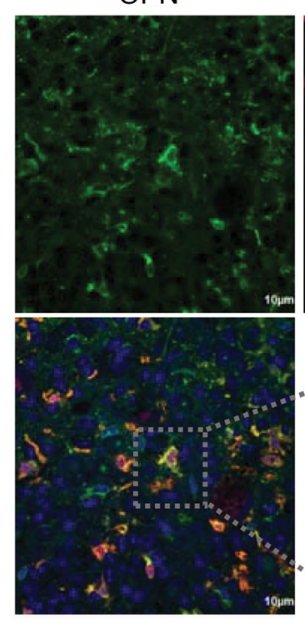

Merge
Iba-1

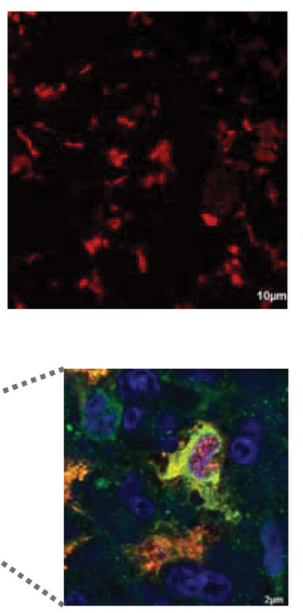

Enlarge

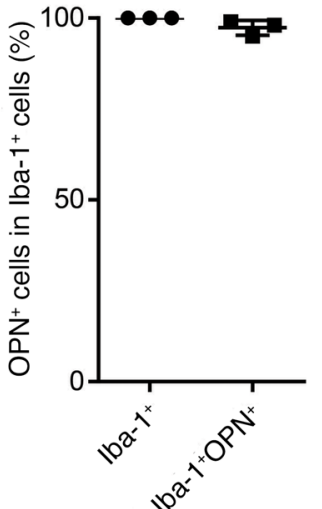

D

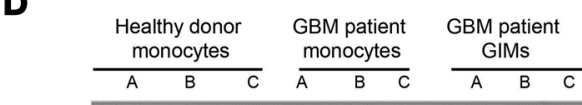

ITGav

ITG 35

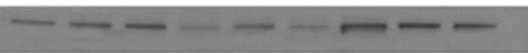

ITG $\beta 3$
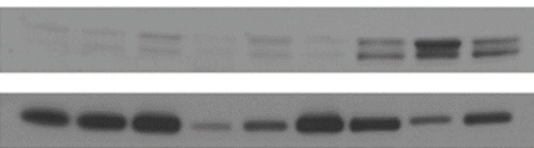

GAPDH
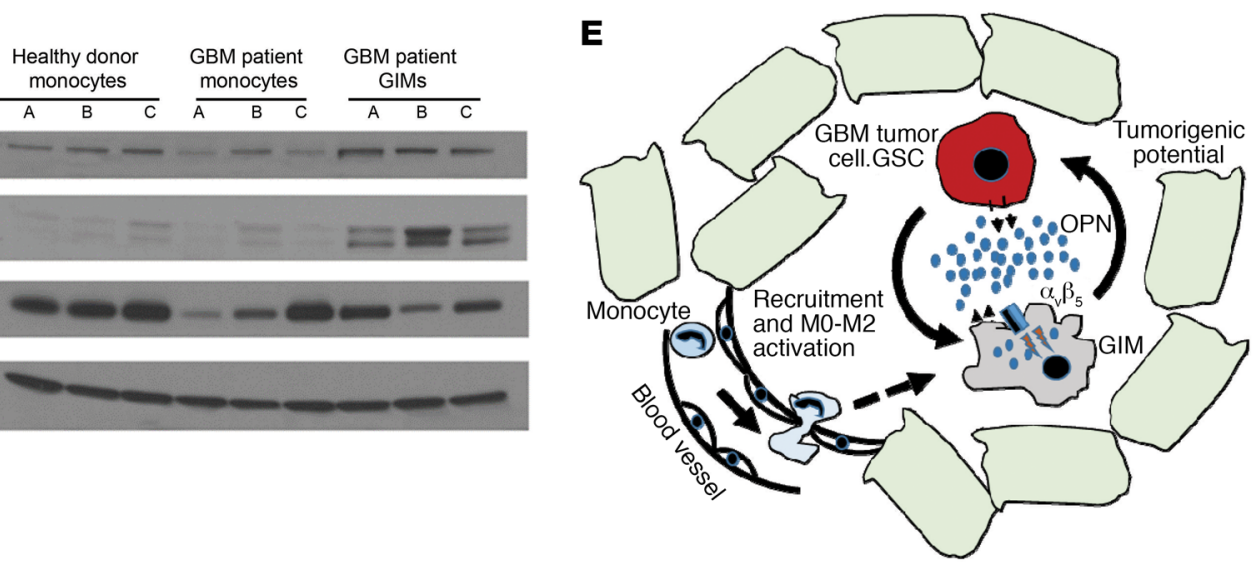
A
MO
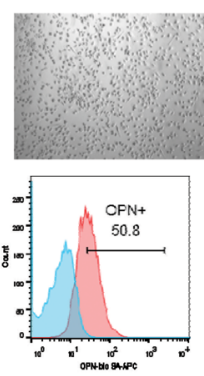

C

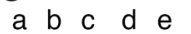

M1
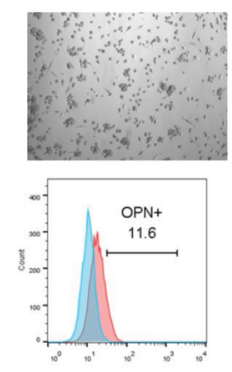

$\mathrm{M} 2$
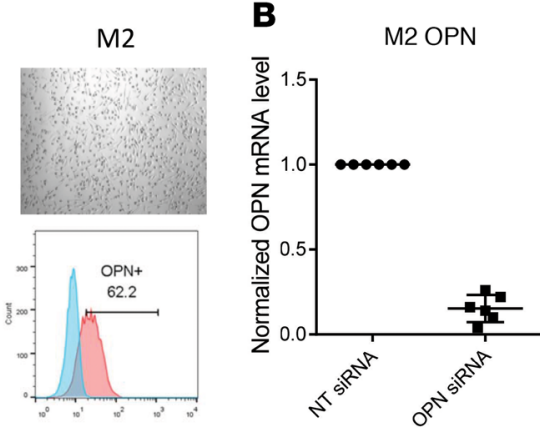

D

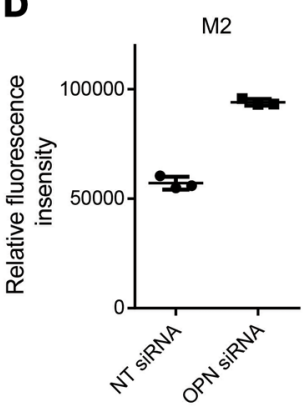

Figure 7. OPN is required for M2 polarization maintenance. (A) CD14 ${ }^{+}$monocytes were polarized, and the M1 and M2 cells were harvested on day 7. Typical phenotypic characteristics of polarized macrophages are shown in the top row. Original magnification, $\times 20$. Flow cytometry data demonstrate that OPN is most abundant in the M2-polarized macrophage (bottom row). Isotype is the shaded blue curve, and OPN staining is shaded red. Representative FACS data from 3 independent experiments are shown. (B) Quantitative PCR to measure OPN mRNA levels in M2 macrophages 2 days after transfection of OPN or NT siRNAs. Data are shown as mean \pm SD from 6 different donors. (C) Significantly downregulated genes in OPN siRNA-transfected M2 macrophages, as determined by transforming the associated NanoString data to fit the normal distribution and analyzing the fold changes for the genes with $P$ values less than 0.05 by Wilcoxon's signed-rank test. Data are from 6 different donors (a-f). (D) M2 phagocytic activity based on the uptake of pHrodo Red BioParticles conjugate in triplicate assays. Data are shown as mean \pm SD and are representative of 3 independent experiments. $P<0.05$ by 2 -tailed 2 -sample $t$ test. greatest extent in GIMs relative to the same patient's peripheral monocytes, as well as to healthy donor peripheral monocytes. GBM tumor cells including GSCs elaborate OPN into the local microenvironment, where it acts as a chemokine for tumor-supportive monocytes and macrophages (i.e., MO and M2). The important biological role of OPN expression in non-tumor-derived cells was further supported by our finding that OPN-deficient mice intracerebrally implanted with GL261 have significantly prolonged survival relative to WT mice. This OPN deficiency was associated with reduced immunesuppressive M2 macrophages, especially within the local tumor microenvironment, and markedly enhanced immune antitumor effector function in both $\mathrm{CD}^{+}$and $\mathrm{CD} 8^{+} \mathrm{T}$ cells in the spleen, blood, and brain tumor. These effects on $\mathrm{T}$ cells could be directly mediated by the M2 macrophage (35-37) or by other yet-undiscovered mechanisms, such as modulation of Tregs. Interestingly, the association of OPN deficiency with Tregs accumulating at metastatic sites has been made previously (18), and our findings provide further evidence that OPN modulates multiple mechanisms of tumor-mediated immune suppression. Finally, our ex vivo mechanistic data demonstrate that GIMs are is not specific to the glioma was not (Figure 8C). Furthermore, the 4-1BB-OPN, but not the 4-1BB-PSMA aptamer, conjugate could be visualized in the glioma tissue with an Alexa Fluor 647 fluorophore (Supplemental Figure 11B). As expected, the 4-1BB-OPN aptamer was associated with enhanced $\mathrm{CD}^{+} \mathrm{T}$ cell immune surveillance within the tumor microenvironment (Figure 8D), specifically both the $\mathrm{CD} 4^{+}$and $\mathrm{CD} 8^{+} \mathrm{T}$ cell populations (Supplemental Figure 11C), but not macrophage infiltration (data not shown). All these data reinforce the premise that the soluble OPN is being exploited as a homing molecule to the tumor microenvironment as opposed to being a direct therapeutic target.

\section{Discussion}

Our data cumulatively suggest a unifying, cohesive mechanism underlying the interplay between tumor cells and the innate immune system. From our in silico analysis of TCGA data sets, OPN had substantial predictive potential in estimating survival in GBM patients and was associated with the mesenchymal subtype - known to be enriched with polarized macrophages. Additionally, OPN expression levels directly correlated with multiple macrophage markers in GBM specimens. Based on our array data, OPN was the molecule that was upregulated to the integrin $\alpha_{\mathrm{v}} \beta_{5}$ pathway.

OPN-mediated chemokine activity of macrophages depends on the interaction of OPN with integrin $\alpha_{v} \beta_{5}$. Although OPN has previously been shown to induce macrophage migration via CD44 (38), our data indicate that cancers utilize additional or alternative chemokine modalities. Intracellular OPN can work as an adaptor to mediate CD44-dependent chemotaxis by chemoattractants such as CSF-1 and RANKL. Secretory OPN can function in a paracrine mode to accelerate lung metastasis by enhancing CCL2 signaling (39). In the current study, we investigated the migration mechanisms of secretory OPN rather than its intracellular form. There are multiple integrins, including $\alpha_{v} \beta_{3}, \alpha_{v} \beta_{5}, \alpha_{v} \beta_{1}$, and $\alpha_{9} \beta_{1}$, that can bind to OPN and mediate subsequent downstream events. OPN has multiple effects in different cell types, with distinct outcomes for disease phenotypes because of these varied receptors. For instance, OPN can promote melanoma growth and angiogenesis via integrin $\alpha_{9} \beta_{1}$ signaling (20). Here, we are the first group to our knowledge to determine that $\alpha_{v} \beta_{5}$ integrin is the major receptor on GIMs, and we have shown that OPN mediates chemoattractive activity for the recruitment and enrichment of these cells into the GBM. From a therapeutic perspective, enriched in the tumor microenviroment through the OPN/ 
A

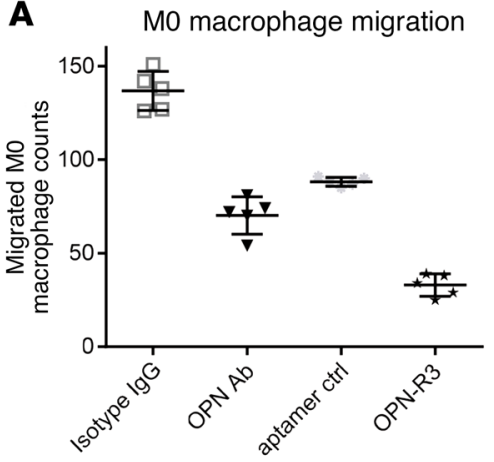

M2 macrophage migration

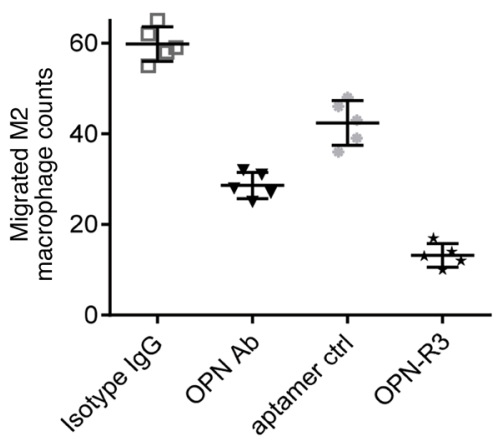

D

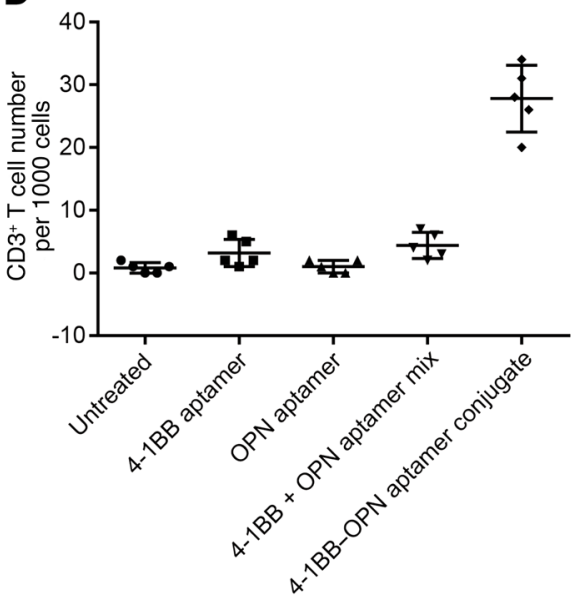

C
B

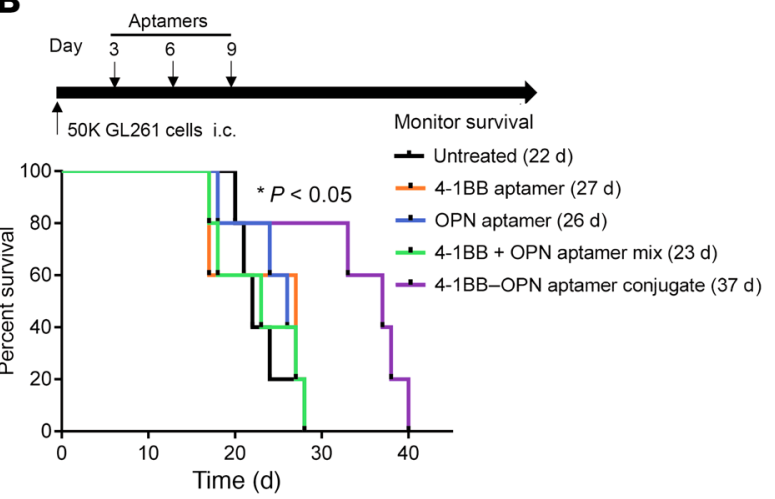

4-1BB-OPN aptamer

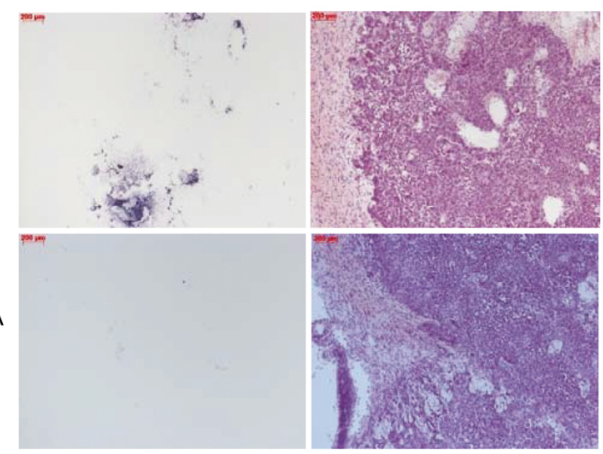

Figure 8. OPN expression can be exploited for therapeutic targeting. (A) Transwell migration assays of MO- and M2-skewed macrophages exposed to OPN antibody or antagonistic OPN aptamers (OPN-R3) at 48 hours. Data are shown as mean \pm SD and are representative of 3 independent experiments. (B) Treatment schema and graph of the Kaplan-Meier survival estimate demonstrating improved survival with the 4-1BB-OPN aptamer conjugate (37 days) relative to the untreated control (22 days), 4-1BB aptamer (27 days), OPN aptamer (26 days), or a mixture of the 4-1BB and OPN aptamers (23 days) ( $n=5$ mice/group) $(P<0.05)$. Data represent 2 independent experiments. i.c., intracerebrally. (C) Localization of the 4-1BB-OPN aptamer in the brain tumor microenvironment visualized with an antisense 4-1BB RNA probe using ISH. A control aptamer that does not target the glioma, 4-1BB-PSMA, was not detected in the brain tumor using the 4-1BB RNA probe. Right panels: Colocalizing H\&E staining of the tumor within the brain. Original magnification, $\times 100$ (scale bars: $200 \mu \mathrm{m}$ ). (D) Summary graph of the treatment groups from $\mathbf{B}$ showing quantification of $\mathrm{CD}^{+} \mathrm{T}$ cells within the intracerebral glioma (4-1BB-OPN aptamer conjugate relative to other groups; $P<0.05$ ). Data indicate mean \pm SD from 5 different animals per group in a single experiment. $P$ values were calculated based on the 2 -tailed 2 -sample $t$ test.
OPN inhibitors/antagonists (such as OPN-specific antibodies and aptamers) and some reagents that specifically block these integrins/CD44 (such as their antibodies and RGD motif peptides) could be considered as potential agents for treating the cancer and other types of diseases in which there is overactive OPN signaling. In addition, targeting the upstream modulators of OPN signaling is another possible strategy to inhibit OPN. Fam20C has recently been identified as an upstream kinase responsible for OPN phosphorylation that is crucial for its physiological functions (40).

In direct contrast to our finding that the $\mathrm{Opn}^{-/-}$background confers enhanced survival in mice bearing intracerebral GL261 tumors, another group has found that there was no increase in survival (41). This discrepancy is probably secondary to the differences in OPN expression in the GL261 glioma cell lines. In the earlier study the GL261 cell line had very low levels of OPN expression, whereas in the current study the GL261 line had high expression of OPN, similar to that seen in human GBM, glioma cell lines, and GSCs. Szulzewsky et al. specifically selected a GL261 line with low levels of OPN expression in order to investigate the effect of OPN ablation in a system where OPN is predominantly derived from the microenvironment.

OPN blockade with an antagonistic aptamer or antibody has been shown to be efficacious in inhibiting breast and lung cancer progression and prolonging survival $(19,22)$. However, no therapeutic effect was observed with these treatment modalities in the GL261 glioma syngeneic mouse model. This ineffectiveness may be due to the failure of the OPN aptamer or antibody to be well internalized in order to block intracellular OPN or gene expression, especially in the setting of CNS tumors protected by the blood-brain barrier. This is supported by our OPN mRNA in situ hybridization (ISH) data showing that OPN expression was not altered in the 
glioma from mice treated with the OPN aptamer. Small-structured single-stranded nucleic acid aptamers have been used as delivery molecules to target distinct cell types, thereby reducing off-target effects or other unwanted side effects. In addition, aptamers have notable advantages over antibody-based therapies: (a) a cell-free and cost-effective manufacturing process; and (b) reduced doselimiting autoimmune toxicities, because small nucleic acids are less likely to trigger or initiate this nonspecific immune response. Use of OPN-targeted bispecific aptamer immune therapeutics may be a broadly applicable and clinically feasible approach to potentiate naturally occurring antitumor immunity via tumor targeting. Moreover, this offers unmatched versatility and feasibility for development and manufacture that could rival, if not replace, antibodies as the platform of immune checkpoint blockade, which are currently being used in multiple clinical trials for cancer patients. Due to its high specificity for targeting immune cells and tumor cells in the GBM tumor microenvironment, use the bispecific 4-1BB-OPN aptamer is a promising therapeutic strategy for patients with GBMs of the mesenchymal subtype.

\section{Methods}

Supplemental Methods are available online with this article.

TCGA. We analyzed publicly available clinical data on glioblastoma patients and their OPN mRNA, which were downloaded from TCGA (https://cancergenome.nih.gov/). Level 3 Illumina RNA-Seq was used for mRNA. For each gene of interest, a relationship with survival was evaluated. Patients were grouped into percentiles according to their OPN mRNA expression.

Isolation of GIMs and peripheral blood monocytes and their gene microarray. Under protocol LAB03-0687, approved by MD Anderson's institutional review board, patients' glioblastomas were graded pathologically according to the WHO classification and were subsequently processed. Peripheral blood was drawn from the patients intraoperatively. The tumor was digested with Liberase TM enzyme, and the myelin was removed by centrifugation with a Percoll gradient, as we have previously described (42). Afterward, the cells were blocked for nonspecific binding using an Fc $\gamma$ R-Binding Inhibitor (Miltenyi Biotec) and were coincubated with magnetically labeled CD11b or CD14 MicroBeads (Miltenyi Biotec) at $4^{\circ} \mathrm{C}$ for 30 minutes. The cell suspension was loaded onto a MACS Column (Miltenyi Biotec) in a magnetic field. The negative fraction was discharged. Upon removal of the column from the MACS separation system, CD11b ${ }^{+}$or $\mathrm{CD} 14^{+}$cells were eluted as a positive fraction. RNA was obtained using the mirVana kit (Ambion), and samples were checked for purity and quality via an Agilent Bioanalyzer before being submitted for human whole-genome microarray analysis (30,275 human genes) provided by the Phalanx Biotech Group. These microarray data were deposited in the NCBI's Genomic Expression Omnibus database (GEO GSE51332). The results of the analysis were used to determine which mRNAs had significant fold differences in expression of GIMs relative to patient-matched monocytes and GIMs relative to normal donor monocytes.

OPN KO by siRNA, shRNA, and CRISPR/Cas 9 technologies. To silence OPN expression, $\mathrm{MO}$ and $\mathrm{M} 2$ macrophages were transfected with human OPN siRNAs and their NT scramble control from Dharmacon using Lipofectamine 2000 (Invitrogen, Life Technologies) according to the manufacturer's instructions. In addition, a CRISPR/Cas9 approach was utilized to knock out OPN expression in GSCs. Briefly, pSpCas9 BB-2A-
GFP (PX458, GenScript) plasmids for expression of 2 sgRNA sequences specific to the OPN exome genome (sgRNA targeting sequences: ATCAGAGTCGTTCGAGTCAA and TGGCTTTCGTTGGACTTACT) as well as an NT PX458 plasmid (sgRNA targeting sequence: CGCTTCCGCGGCCCGTTCAA) were designed by a member of our research team and incorporated into PX458 (at GenScript). The PX458 plasmids ( $2 \mu \mathrm{g}$ each) were then transfected into the GSCs using the Amaxa mouse NSC Nucleofector kit (program A-033, Lonza). On day 4 after electroporation, $\mathrm{GFP}^{+}$cells were sorted and seeded as single cells into 96-well plates by a FACSAria Sorter (BD Biosciences). Once single-cell colonies grew out, a targeted region of the Opn gene was PCR amplified from the genomic DNA of each clone. The products were purified with a PCR clean-up kit (QIAGEN) and then sequenced by using the same set of PCR primers (forward primer: TTCAATGGGCAGTTTTGAGC; reverse primer: ACTTACAAAAACCGCCAAGC) in order to screen for Opn gene-deficient clones. The complete absence of OPN expression was verified in the GSC-conditioned medium (Supplemental Figure 3). Last, murine and human OPN shRNA BLOCK-iTTM lentiviral vectors were purchased from Thermo Fisher Scientific, and GL261 cells and GSCs were infected with their packaged OPN and scramble shRNA lentiviruses. Blasticidin $(10 \mu \mathrm{g} / \mathrm{ml})$ was added 24 hours after infection. Resistant clones were collected after 2 weeks, numerically expanded, and analyzed for OPN mRNA expression by quantitative PCR.

Macrophage migration assay. Transwell assays assessing cell migration were performed in 24 -well plates with inserts $(8-\mu \mathrm{m}$ pore size, BD Biosciences). Briefly, $5 \times 10^{4}$ macrophages were seeded in the upper chamber precoated with Matrigel (BD Biosciences). The lower chamber was filled with either medium containing $10 \% \mathrm{FBS}$ as a positive control or serum-free medium containing $0.1 \%$ BSA as a negative control. Cells were allowed to invade or migrate for 48 hours before trypan blue staining. The supernatants from GSCs were treated with $10 \mu \mathrm{g} / \mathrm{ml}$ OPN-neutralizing antibody (R\&D Systems) for 2 hours at $37^{\circ} \mathrm{C}$ and then applied to the lower chamber to attract macrophages. Supernatants from OPN sgRNA/CRISPR (designated OPN KO) and NT scramble GSC lines (designated NT ctrl) were also included. Recombinant human OPN protein (10 ng/ml in most conditions) and its neutralizing antibody as well as integrin $\alpha_{\mathrm{v}} \beta_{5}$ antibody $(10 \mu \mathrm{g} / \mathrm{ml})$ were purchased from R\&D Systems, and recombinant human CCL2 (10 ng/ml) was from Peprotech.

OPN neutralizing antibody and aptamers. Anti-murine OPN neutralizing antibody was produced by the hybridoma cell line clone MPIIIB10(1) obtained from the Developmental Studies Hybridoma Bank. Briefly, 21 RPMI 1640 culture supernatant were collected during log-phase cell growth, and the antibody was purified with a Protein G column (GE Healthcare). The OPN-R3 aptamer was synthesized by Sigma-Aldrich, and its sequence was $5^{\prime}$-CGGCCACAGAAUGAAAAACCUCAUCGAUGUUGCAUAGUUG-3' (2'-O-methylation for all $\mathrm{C}$ and $\mathrm{U}$ nucleotides). The PSMA aptamer served as a nonspecific control for the OPN aptamer when conjugated to the 4-1BB aptamer, and its sequence was: $5^{\prime}$-GGGAGGACGAUGCGGAUCAGCCAUGUUUACGUCACUCCU-3' (2'-O-methylation for all $\mathrm{C}$ and $\mathrm{U}$ nucleotides). The 4-1BB aptamer sequence was 5'-GGGCGGGAGAGAGGAAGAGGGAUGGGCGACCGAACGUGCCCUUCAAAGCCGUUCACUAACCAGUGGCAUAACCCAGAGGUCGAUAGUACUGGAUCCCGCCCUCCUGCGGCCGAGAGAGGAAGAGGGAUGGGCGACCGAACGUGCCCUUCAAAGCCGUUCACUAACCAGUGGCAUAACCCAGAGGUCGAUAGUACUG- 
GAUCGGCCGCUCCCG-3'; and the linker sequence between the 4 -1BB aptamer and the OPN or PSMA aptamer was 5'-CUAUAAGUGUGCAUGAGAAC-3'. Alexa Fluor 647-labeled OPN and 4-1BBPSMA aptamers were synthesized by Integrated DNA Technologies and were used for tracking localization into the brain tumors. The aptamer conjugates were generated by annealing equal amounts of Alexa Fluor 647-labeled OPN or PSMA aptamer to the 4-1BB aptamer. The OPN aptamer, 4-1BB aptamer, and 4-1BB-OPN bispecific aptamers for in vivo study have been previously described (43).

Animalmodels. OPN-deficient mice (Opn ${ }^{-/}$, B6.Cg-Spp1tm1blh/J, congeneic with C57BL/6J) were purchased from the Jackson Laboratory. Progeny were PCR genotyped for verification of Opn gene deficiency. WT C57BL/6J mice were obtained from the Experimental Radiation Oncology in-house breeding facility at MD Anderson. Mice were maintained in the MD Anderson Isolation Facility in accordance with Laboratory Animal Resources Commission standards and handled according to approved protocol 08-06-11831.

4-1BB aptamer and OPN mRNA probe preparation for ISH. In order to make a DNA template for the 4-1BB aptamer, a T7 RNA polymerase promoter sequence (5'-CCCTATAGTGAGTCGTATTACGC- $3^{\prime}$ ) was added to the $3^{\prime}$ end and SP6 promoter sequence (5'-GCGATTTAGGTGACACTATAG $-3^{\prime}$ ) to the $5^{\prime}$ end of the $4-1 \mathrm{BB}$ aptamer DNA double-strand sequence. This whole sequence was synthesized and cloned into the pUC57 plasmid with EcoRI and HindIII at its 5' and 3 ' ends, respectively. Upon sequencing confirming that the insert and flanking sequences were correct, the plasmid was linearized with EcoRI (NEB) to generate the 4-1BB aptamer DNA template with the T7 promoter. Linearization with HindIII (NEB) generated a nonspecific antisense DNA template with the SP6 promoter. These were subsequently transcribed into RNA probes by RNA polymerase (NEB) to detect the $4-1 \mathrm{BB}-\mathrm{OPN}$ or $4-1 \mathrm{BB}-\mathrm{PSMA}$ aptamer within the gliomas using ISH. To generate the OPN mRNA probe, mouse Opn gene exon 8 ( $869 \mathrm{bp}$ ) was synthesized by GenScript, and this DNA template was then transcribed into an RNA probe to detect Opn gene expression in the glioma. The RNA probes were then labeled with digoxigenin for use in the glioma ISH (DIG RNA labeling kit, Sigma-Aldrich).

Statistics. For survival analysis using TCGA database, the log-rank test was used to determine the association between mRNA expression and overall survival. The Kaplan-Meier method was used to generate survival curves. Data are presented as mean \pm SD or mean \pm SEM, and all in vitro experiments were replicated 3 times (except as noted in the figure legends). Differences between groups were analyzed using a 1-way ANOVA test or an unpaired 2-tailed $t$ test. Bonferroni's correction was applied to correct for multiple comparisons. $P<0.05$ was considered statistically significant.

Study approval. Patient studies were performed under protocol LAB03-0687, approved by the MD Anderson Institutional review board. The written informed consent from each patient was received. The mice used in this study were maintained according to the NIH
Guide for the Care and Use of Laboratory Animals (National Academies Press, 2011); maintained in the MD Anderson Isolation Facility in accordance with Laboratory Animal Resources Commission standards; and handled and euthanized according to procedures (no. 08-06-11831) approved by the IACUC of the University of Texas MD Anderson Cancer Center.

\section{Author contributions}

JW conceived, directed, and performed the experiments; analyzed and interpreted experimental data; and wrote the manuscript. AM performed experiments in shRNA cell line construction, migration assays, ELISA, and in vivo survival studies, and edited the manuscript. BS synthesized and modified aptamers, conceived aptamer in vivo tracking experiments, and edited the manuscript. KG performed Western blotting, constructed the polarized macrophage gene library, and edited the manuscript. YH performed intracranial tumor implantation and the in vivo aptamer therapeutics study. MO performed intracranial tumor implantation and OPN-treated macrophage phenotyping, and edited the manuscript. ZG performed CRISPR/Cas9 GSC line selection, and analyzed and interpreted experimental data. LYK performed intracranial tumor implantation. XL performed histochemistry staining. HC conceived of the $\mathrm{T}$ cell cytotoxicity experiments and edited the manuscript. SZ performed statistical analysis for all experiments. YAW, GNF, and JH conceived experiments and edited the manuscript. EG conceived the in vivo therapeutic study for the aptamers and edited the manuscript. NK and XH performed construction and selection of CRISPR/Cas9 plasmids, and edited the manuscript. RV analyzed and interpreted OPN expression data downloaded from TCGA. SL conceived experiments and edited the manuscript. ABH conceived the study, directed experiments, interpreted experimental data, and wrote the manuscript.

\section{Acknowledgments}

The authors acknowledge the Flow Cytometry and Cellular Imaging Core Facility at MD Anderson, which is funded by the National Cancer Institute (CA16672), for assistance with flow cytometry data acquisition. This project was supported by the RNA In Situ Hybridization Core at Baylor College of Medicine, which is partly supported by a Shared Instrumentation grant from the NIH (1S10OD016167). Special thanks go to David M. Wildrick and Audria Patrick for editorial and administrative support. This study was supported by the Ben and Catherine Ivy Foundation, the Provost Retention Fund, and NIH grants CA120895, P50CA127001, and P30CA016672.

Address correspondence to: Amy B. Heimberger, Department of Neurosurgery, The University of Texas MD Anderson Cancer Center, Unit 422, P.O. Box 301402, Houston, Texas 77230-1402, USA. Phone: 713.792.2400; Email aheimber@mdanderson.org.
1. Alexander BM, Cloughesy TF. Adult glioblastoma. JClin Oncol. 2017;35(21):2402-2409.

2. Wai PY, Kuo PC. The role of osteopontin in tumor metastasis. J Surg Res. 2004;121(2):228-241.

3. Gao C, Guo H, Downey L, Marroquin C, Wei J, Kuo PC. Osteopontin-dependent CD44v6 expression and cell adhesion in HepG2 cells.
Carcinogenesis. 2003;24(12):1871-1878.

4. Rangaswami H, Bulbule A, Kundu GC. Osteopontin: role in cell signaling and cancer progression. Trends Cell Biol. 2006;16(2):79-87.

5. Weber GF, Lett GS, Haubein NC. Osteopontin is a marker for cancer aggressiveness and patient survival. Br JCancer. 2010;103(6):861-869.
6. Toy H, Yavas O, Eren O, Genc M, Yavas C. Correlation between osteopontin protein expression and histological grade of astrocytomas. Pathol Oncol Res. 2009;15(2):203-207.

7. Matusan-Ilijas K, Behrem S, Jonjic N, Zarkovic K, Lucin K. Osteopontin expression correlates with angiogenesis and survival in malignant astrocy- 
toma. Pathol Oncol Res. 2008;14(3):293-298.

8. Schuhmann MU, et al. Peptide screening of cerebrospinal fluid in patients with glioblastoma multiforme. Eur J Surg Oncol. 2010;36(2):201-207.

9. Sreekanthreddy P, et al. Identification of potential serum biomarkers of glioblastoma: serum osteopontin levels correlate with poor prognosis. Cancer Epidemiol Biomarkers Prev. 2010;19(6):1409-1422.

10. Jan $\mathrm{HJ}$, et al. Osteopontin regulates human glioma cell invasiveness and tumor growth in mice. Neuro-oncology. 2010;12(1):58-70.

11. Lu DY, Yeh WL, Huang SM, Tang CH, Lin HY, Chou SJ. Osteopontin increases heme oxygenase-1 expression and subsequently induces cell migration and invasion in glioma cells. Neuro-oncology. 2012;14(11):1367-1378.

12. Yan W, et al. Expression pattern of osteopontin splice variants and its functions on cell apoptosis and invasion in glioma cells. Neuro-oncology. 2010;12(8):765-775.

13. Pietras A, et al. Osteopontin-CD44 signaling in the glioma perivascular niche enhances cancer stem cell phenotypes and promotes aggressive tumor growth. Cell Stem Cell. 2014;14(3):357-369.

14. Lamour V, et al. Selective osteopontin knockdown exerts anti-tumoral activity in a human glioblastoma model. Int J Cancer. 2010;126(8):1797-1805.

15. Cantor H, Shinohara ML. Regulation of T-helpercell lineage development by osteopontin: the inside story. Nat Rev Immunol. 2009;9(2):137-141.

16. Renkl AC, et al. Osteopontin functionally activates dendritic cells and induces their differentiation toward a Th1-polarizing phenotype. Blood. 2005;106(3):946-955.

17. Atai NA, et al. Osteopontin is up-regulated and associated with neutrophil and macrophage infiltration in glioblastoma. Immunology. 2011;132(1):39-48.

18. Sangaletti S, et al. Osteopontin shapes immunosuppression in the metastatic niche. Cancer Res. 2014;74(17):4706-4719.

19. Kim EK, et al. Tumor-derived osteopontin suppresses antitumor immunity by promoting extramedullary myelopoiesis. Cancer Res. 2014;74(22):6705-6716.

20. Kale S, Raja R, Thorat D, Soundararajan G, Patil TV, Kundu GC. Osteopontin signaling upregulates cyclooxygenase-2 expression in tumor-associated macrophages leading to enhanced angiogenesis and melanoma growth via $\alpha 9 \beta 1$ integrin. Oncogene. 2014;33(18):2295-2306.

21. Schrand B, Berezhnoy A, Brenneman R, Williams A, Levay A, Gilboa E. Reducing toxicity of 4-1BB costimulation: targeting 4-1BB ligands to the tumor stroma with bi-specific aptamer conjugates. Oncoimmunology. 2015;4(3):e970918.

22. Mi Z, Guo H, Russell MB, Liu Y, Sullenger BA, Kuo PC. RNA aptamer blockade of osteopontin inhibits growth and metastasis of MDA-MB231 breast cancer cells. Mol Ther. 2009;17(1):153-161.

23. Talbot LJ, Mi Z, Bhattacharya SD, Kim V, Guo $\mathrm{H}$, Kuo PC. Pharmacokinetic characterization of an RNA aptamer against osteopontin and demonstration of in vivo efficacy in reversing growth of human breast cancer cells. Surgery. 2011;150(2):224-230.

24. Doucette T, et al. Immune heterogeneity of glioblastoma subtypes: extrapolation from the cancer genome atlas. Cancer Immunol Res. 2013;1(2):112-122.

25. Wang $\mathrm{Q}$, et al. Tumor evolution of glioma-intrinsic gene expression subtypes associates with immunological changes in the microenvironment. Cancer Cell. 2017;32(1):42-56.e6.

26. Weber GF, Zawaideh S, Hikita S, Kumar VA, Cantor H, Ashkar S. Phosphorylation-dependent interaction of osteopontin with its receptors regulates macrophage migration and activation. JLeukoc Biol. 2002;72(4):752-761.

27. Wei J, et al. Glioblastoma cancer-initiating cells inhibit T-cell proliferation and effector responses by the signal transducers and activators of transcription 3 pathway. Mol Cancer Ther. 2010;9(1):67-78.

28. Wei J, et al. miR-124 inhibits STAT3 signaling to enhance $\mathrm{T}$ cell-mediated immune clearance of glioma. Cancer Res. 2013;73(13):3913-3926.

29. Gabrusiewicz K, et al. Glioblastoma-infiltrated innate immune cells resemble $\mathrm{MO}$ macrophage phenotype. JCI Insight. 2016;1(2): e85841.

30. Krause SW, Rehli M, Kreutz M, Schwarzfischer L, Paulauskis JD, Andreesen R. Differential screening identifies genetic markers of monocyte to macrophage maturation. J Leukoc Biol. 1996;60(4):540-545.

31. Bruemmer D, et al. Angiotensin II-accelerated atherosclerosis and aneurysm formation is attenuated in osteopontin-deficient mice. JClin Invest.
2003;112(9):1318-1331.

32. Nyström T, Dunér P, Hultgårdh-Nilsson A. A constitutive endogenous osteopontin production is important for macrophage function and differentiation. Exp Cell Res. 2007;313(6):1149-1160.

33. Wu A, et al. Glioma cancer stem cells induce immunosuppressive macrophages/microglia. Neuro-oncology. 2010;12(11):1113-1125.

34. Laderach D, Movassagh M, Johnson A, Mittler RS, Galy A. 4-1BB co-stimulation enhances human $\mathrm{CD} 8(+) \mathrm{T}$ cell priming by augmenting the proliferation and survival of effector CD8(+) T cells. Int Immunol. 2002;14(10):1155-1167.

35. Gelderman KA, et al. Macrophages suppress T cell responses and arthritis development in mice by producing reactive oxygen species. JClin Invest. 2007;117(10):3020-3028.

36. Huber S, Hoffmann R, Muskens F, Voehringer D. Alternatively activated macrophages inhibit T-cell proliferation by Stat6-dependent expression of PD-L2. Blood. 2010;116(17):3311-3320.

37. Ruffell B, et al. Macrophage IL-10 blocks CD8+ $\mathrm{T}$ cell-dependent responses to chemotherapy by suppressing IL-12 expression in intratumoral dendritic cells. Cancer Cell. 2014;26(5):623-637.

38. Zhu B, et al. Osteopontin modulates CD44dependent chemotaxis of peritoneal macrophages through G-protein-coupled receptors: evidence of a role for an intracellular form of osteopontin. JCell Physiol. 2004;198(1):155-167.

39. Giopanou I, et al. Tumor-derived osteopontin isoforms cooperate with TRP53 and CCL2 to promote lung metastasis. Oncoimmunology. 2017;6(1):e1256528.

40. Tagliabracci VS, et al. A single kinase generates the majority of the secreted phosphoproteome. Cell.2015;161(7):1619-1632.

41. Szulzewsky F, et al. Loss of host-derived osteopontin creates a glioblastomapromoting microenvironment. Neuro-oncology. 2018;20(3):355-366.

42. Hussain SF, Yang D, Suki D, Grimm E, Heimberger AB. Innate immune functions of microglia isolated from human glioma patients. J Transl Med. 2006;4:15.

43. Schrand B, et al. Targeting 4-1BB costimulation to the tumor stroma with bispecific aptamer conjugates enhances the therapeutic index of tumor immunotherapy. Cancer Immunol Res. 2014;2(9):867-877. 